UNITED STATES DEPARTMENT OF THE INTERIOR

GEOLOGICAL SURVEY

\title{
Analytical results and sample locality map \\ for stream-sediment and panned-concentrate samples \\ from the Lime Canyon and Million Hills \\ Wilderness Study Areas, Clark County, Nevada
}

\begin{abstract}
By
John B. McHugh ${ }^{\star}$, John H. Bullock, Jr. ${ }^{\star}$, Theodore A. Roemer ${ }^{\star}$,

Paul H. Briggs ${ }^{\star}$, and Gary A. Nowlan ${ }^{\star}$
\end{abstract}

Open-File Report $89-25$

This report is preliminary and has not been reviewed for conformity with U.S. Geological Survey editorial standards and stratigraphic nomenclature. Any use of trade names is for descriptive purposes only and does not imply endorsement by the USGS.

*U.S. Geological Survey, DFC, Box 25046, MS 973, Denver, CO 80225 


\section{CONTENTS}

Page

Studies Related to Wilderness.................................. 1

Introduction............................................. 1

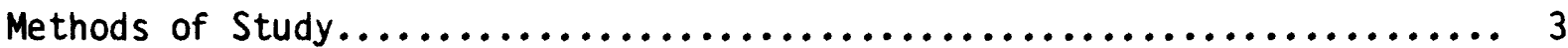

Sample Media............................................ 3

Sample Collection and Preparation............................ 3

Stream-sediment samples............................. 4

Nonmagnetic heavy-mineral-concentrate samples............. 4

Raw panned-concentrate samples......................... 4

Sample Analysis....................................... 4

Spectrographic method................................ 4

Other methods...................................... 5

Data Storage System....................................... 5

Description of Data Tables................................... 5

References Cited.............................................. 6

\section{ILLUSTRATIONS}

Figure 1. Index map of the Lime Canyon and Million Hills

Wilderness Study Areas, Clark County, Nevada................... 2

Plate 1. Sampling sites for stream-sediment and panned-concentrate samples, Lime Canyon and Million Hills Wilderness Study Areas,

Clark County, Nevada................................. in pocket

\section{TABLES}

Table 1. Limits of determination for the spectrographic analysis of stream-sediment samples.................................... 7

Table 2. Limits of determination for the spectrographic analysis of nonmagnet ic heavy-mineral-concentrate samples................. 8

Table 3. Analytical methods used other than emission spectrography...... 9

Table 4. Results of analyses of stream-sediment samples............. 10

Table 5. Results of analyses of heavy-mineral-concentrate samples....... 15

Table 6. Results of analyses of raw panned-concentrate samples......... 20 


\section{STUDIES RELATED TO WILDERNESS}

\section{Bureau of Land Management Wilderness Study Areas}

The Federal Land Policy and Management Act (Public Law 94-579, October 21, 1976) requires the U.S. Geological Survey and the U.S. Bureau of Mines to conduct mineral surveys on certain areas to determine the ir mineral resource potential. Results must be made available to the public and be submitted to the President and the Congress. This report presents the results of geochemical surveys of the Lime Canyon (NV-050-231) and Million Hills (NV050-233) Wilderness Study Areas, Clark County, Nevada.

\section{INTRODUCTION}

In Apri1 1987, the U.S. Geological Survey conducted reconnaissance geochemical surveys of the Lime Canyon and Million Hills Wilderness Study Areas, Clark County, Nevada. The Lime Canyon and Million Hills Wilderness Study Areas are, respectively, about 45 and $60 \mathrm{mi}$ east of Las Vegas, Nevada (fig. 1). The wilderness study areas are north of Lake Mead and between the Overton Arm of Lake Mead on the west and the Arizona state line on the east.

The Lime Canyon Wilderness Study Area comprises 34,680 acres (54 $\mathrm{mi}^{2}$ ) and the Million Hills Wilderness Study Area comprises 9,599 acres $\left(15 \mathrm{mi}^{2}\right)$. Access to the wilderness study areas is from Interstate 15 near Mesquite, Nevada, by way of Nevada Route 170 and then over paved, gravel, and dirt roads. The north, east, and south boundaries of the Lime Canyon Wilderness Study Area can generally be reached by dirt roads; the west boundary is generally inaccessible by vehicle. The boundary of Million Hills Wilderness Study Area can be reached by vehicle only on the road that follows Garden Wash; elsewhere around Million Hills Wilderness Study Area, roads are from one-half to several miles away.

Elevations range from about $1,500 \mathrm{ft}$ to about $4,400 \mathrm{ft}$ in the Lime Canyon Wilderness Study Area and from about $1,800 \mathrm{ft}$ to about 4,700 ft in the Million Hills Wilderness Study Area. Vegetation in both wilderness study areas is predominately desert shrubs, creosote, cacti, and yucca. Joshua trees are present in parts of the Lime Canyon Wilderness Study Area. Topography of the two wilderness study areas is similar. Both areas have major north-southtrending ridges with outwash plains sloping toward Lake Mead or toward the stream valleys that are tributary to Lake Mead. In the Lime Canyon Wilderness Study Area, the dominant north-south ridge is Lime Ridge and the outwash plain slopes to the west. In the Million Hills Wilderness Study Area, the dominant ridge is Azure Ridge and the outwash plain slopes east.

Longwe 11 and others (1965) described the geology of Clark County. More recent 1y, geology of the Las Vegas $1^{\circ} \times 2^{\circ}$ quadrangle (Bohannon, 1978) and the state of Nevada (Stewart and Carlson, 1978) were compiled; both compilations include the wilderness study areas. A major structural feature near the wilderness study areas is the Gold Butte fault. The two wilderness study areas are on either side of the northeast-trending fault and are separated by about $10 \mathrm{mi}$ along the surface trace of the fault. The Lime Canyon Wilderness Study Area is north of the Gold Butte fault and is west of the Million Hills Wilderness Study Area, which is mostly south of the fault. Bohannon (1979) presents evidence that the Gold Butte fault is a left-lateral strike-slip fault with an offset of about $6 \mathrm{mi}$.

Geology of the two wilderness study areas is quite similar. Precambrian metamorphic rocks underlie relatively small parts of both areas but the 


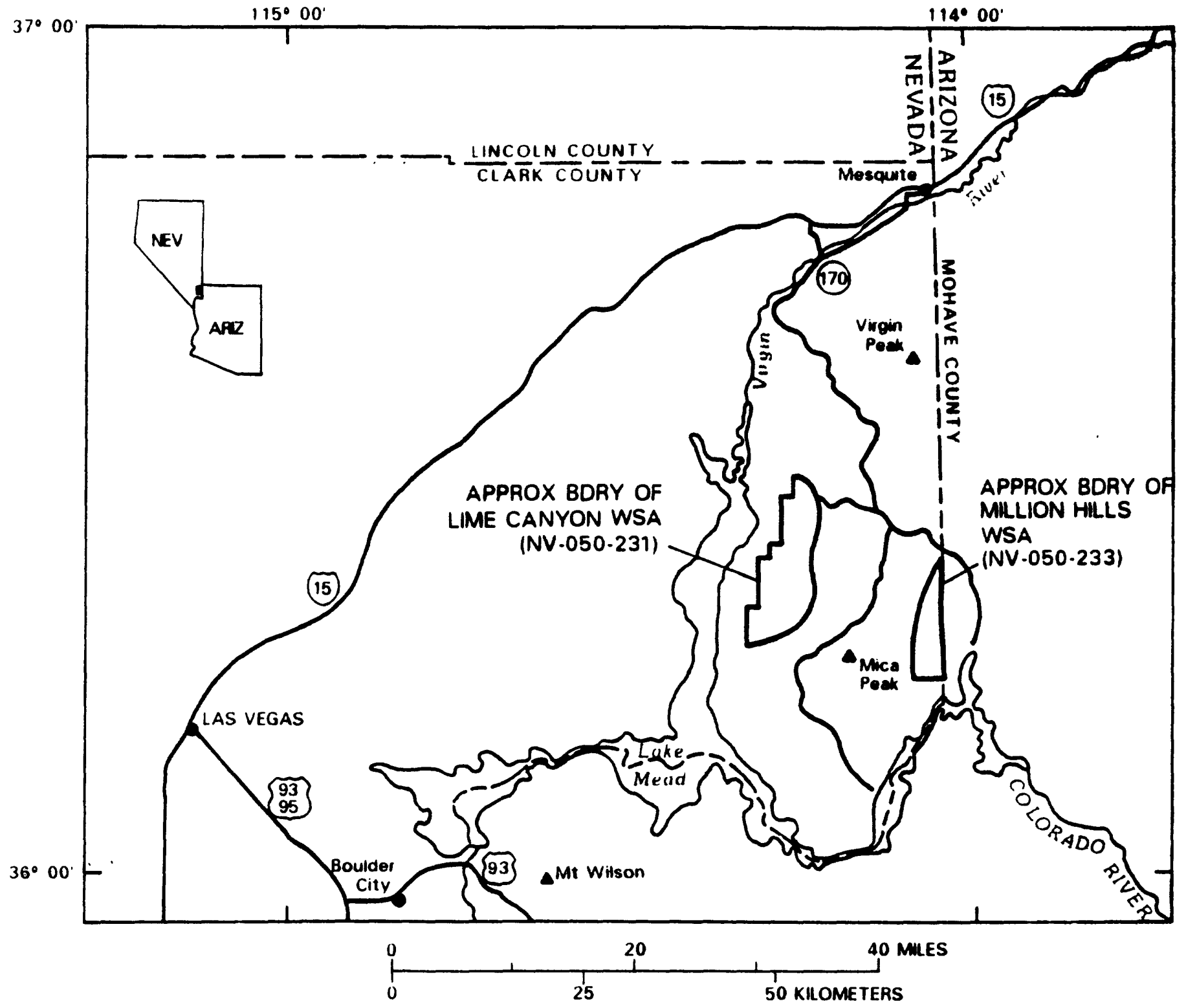

Fiqure 1. Index map of the Lime Canyon and Million Hills Wilderness Study Areas, Clark County, Nevada. 
wilderness study areas are on the margins of extensive areas of these rocks. The major north-south-trending ridges are composed mostly of Paleozoic carbonate rocks. Paleozoic or Mesozoic sandstones and shales also underlie extensive parts of both areas. Tertiary volcanic rocks are present in both areas and Quaternary gravels blanket the outwash plains.

Mineral deposits of Clark County were described by Longwe 11 and others (1965). The Lime Canyon Wilderness Study Area is within the Gold Butte mining district. Mineral deposits and mining activity within and near the Lime Canyon Wilderness Study Area are described by Winters (1988); occurrences with in $2 \mathrm{mi}$ include minor concentrations of gold, silver, copper, lead, and zinc in bedrock and dumps, minor placer gold, patented gypsum claims, and numerous uranium exploration trenches.

The Million Hills Wilderness Study Area is on the east edge of the Gold Butte mining district. Causey (1988) describes mines, prospects, and mineralized areas in and within $0.5 \mathrm{mi}$ of the wilderness study area. The Azure Ridge mine produced several carloads of zinc or copper ore and probably a few thousands of pounds of lead. The Azure Ridge mine is in Cambrian(?) dolomite. Causey (1988) found higher-than-expected cobalt concentrations (as high as $471 \mathrm{ppm}$ ) in brecciated dolomite at the mine. Manganese-rich layers in Quaternary a 17uvium about one-half mile west-northwest of site 151 (plate 1) contain as much as $6,100 \mathrm{ppm}$ cobalt, $1,700 \mathrm{ppm}$ nicke $1,1,900 \mathrm{ppm}$ lead, 3,700 ppm zinc, 500 ppm copper, 100 ppm molybdenum, and 80 ppm thallium (Causey, 1988); the bedrock source of these concentrations is unknown.

\section{METHODS OF STUDY}

\section{Sample Media}

Analyses of stream-sediment samples represent the chemistry of the rock material eroded from the drainage basin upstream from each sample site. Such information is useful in identifying those basins which contain concentrations of elements that may be related to mineral deposits. Panned-concentrate samples derived from stream sediment provide information about the chemistry of certain minerals in rock material eroded from the drainage basin upstream from each sample site. The selective concentration of minerals, many of which may be ore related, permits determination of some elements that are not easily detected in stream-sediment samples.

\section{Sample Collection and Preparation}

Stream-sediment and panned-concentrate samples were collected at 60 sites in and near the Lime Canyon Wilderness Study Area and 28 sites in and near the Million Hi11s Wilderness Study Area (plate 1). Sampling density was about one sample site per $0.9 \mathrm{mi}^{2}$ in the Lime Canyon Wilderness Study Area and one sample site per $0.5 \mathrm{mi}^{2}$ in the Million Hills Wilderness, Study Area. The area of the drainage basins sampled ranged from about $0.1 \mathrm{mi}^{2}$ to about $3 \mathrm{mi}^{2}$.

Two types of panned-concentrate samples were collected at each site. One is termed a "nonmagnetic heavy-mineral-concentrate sample" and the other a "raw panned-concentrate sample." Now 1 an.

Samples were collected by Janet L. Jones, Steven M. Smith, and Gary A. 


\section{Stream-sed iment samples}

The stream-sediment samples consisted of active alluvium collected primarily from first-order (unbranched) and second-order (below the junction of two first-order) streams (plate 1). The stream-sediment samples were air dried, then sieved using 80 -mesh $(0.17-\mathrm{mm})$ stainless-steel sieves. The portion of the sediment passing through the sieve was pulverized to approximately minus 100 -mesh $(0.15-\mathrm{mm})$ for analysis.

\section{Nonmagnetic heavy-mineral-concentrate samples}

Active alluvium was screened with a $2.0-\mathrm{mm}$ (10-mesh) screen to obtain about $20 \mathrm{lb}$ of sample after removal of the coarse material. The samples were then panned to remove most of the quartz, feldspar, carbonate rock material, organic material, and clay-sized material. The resulting concentrate samples weighed an estimated 0.5-3 0z.

After the samples were oven dried at $90{ }^{\circ} \mathrm{C}$, bromoform (specific gravity 2.8) was used to remove the remaining quartz, feldspar, and other light minerals. The resultant heavy-mineral sample was separated into three fractions using a large electromagnet (in this case a modified Frantz Isodynamic Separator). The most magnetic material, primarily magnetite, was not analyzed. The second fraction, largely ferromagnesian silicates and iron oxides, was saved for archival storage. The third fraction (the least magnetic material, which may include the nonmagnetic ore minerals, zircon, sphene, etc.) was split using a Jones splitter. One split was hand ground for spectrographic analysis; the other split was saved for mineralogical analysis. These magnetic separates are the same separates that would be produced by using a Frantz Isodynamic Separator set at a slope of $15^{\circ}$ and a tilt of $10^{\circ}$ with a current of 0.2 ampere to remove the magnetite and ilmenite, and a current of 0.6 ampere to split the remainder of the sample into paramagnetic and nonmagnetic fractions.

\section{Raw panned-concentrate samples}

Active alluvium was screened, panned, and dried in the same manner as the nonmagnetic heavy-mineral-concentrate samples. However, in each case, the entire raw panned-concentrate sample was weighed and analyzed for gold ( $\mathrm{Au}$ ) without further treatment.

\section{Sample Analysis}

\section{Spectrographic method}

The stream-sediment samples were analyzed for 31 elements and the nonmagnetic heavy-mineral-concentrate samples for 37 elements using a semiquantitative, direct-current arc emission spectrographic method (Grimes and Marranzino, 1968). The elements analyzed and their limits of determination are listed in tables 1 and 2 . Spectrographic results were obtained by visual comparison of spectra derived from the sample against spectra obtained from standards made from pure oxides and carbonates. Standard concentrations are geometrically spaced over any given order of magnitude of concentration as follows: 100,50,20,10, and so forth. Samples whose concentrations are estimated to fall between those values are assigned values of $70,30,15$, and so forth. The precision of the analytical 
method is approximately plus or minus one reporting interval at the 83 percent confidence level and plus or minus two reporting intervals at the 96 percent confidence level (Motooka and Grimes, 1976). Emission spectrographic analyses were performed by John H. Bullock, Jr.

\section{Other methods}

Stream-sediment samples were also analyzed by inductively coupled plasmaatomic emission spectroscopy (ICP) and ultraviolet fluorimetry. The samples were analyzed for arsenic (As), antimony ( $\mathrm{Sb}$ ), bismuth (Bi), cadmium (Cd), and zinc ( $Z n)$ using ICP and for uranium (U) using ultraviolet fluorimetry. Stream-sediment and raw panned-concentrate samples were analyzed for gold ( $\mathrm{Au}$ ) by atomic absorption spectroscopy (AA). Limits of determination, precision, and references for the methods are included in table 3 . Analysts were Paul $H$. Briggs, John B. McHugh, and Theodore A. Roemer.

Analytical results for stream-sediment, nonmagnetic heavy-mineralconcentrate, and raw panned-concentrate samples are listed in tables 4, 5, and 6 , respectively.

\section{DATA STORAGE SYSTEM}

Upon completion of the analytical work, the results were entered into a U.S. Geological Survey computer data base called PLUTO. This data base contains both descriptive geological information and analytical data. Any or a) 1 of this information may be retrieved and converted to a binary form (STATPAC, VanTrump and Miesch, 1977) for computerized statistical analysis or publication.

\section{DESCRIPTION OF DATA TABLES}

The numeric part of each sample identification in tables 4-6 is the same as the corresponding sampling-site number on plate 1. A letter " $N$ " in the tables indicates that a given element was looked for but not detected at the lower limit of determination shown for that element in tables 1 and 2 . For emission spectrographic analyses, a "less than" symbol $(<)$ entered in the tables indicates that an element was observed but was below the lowest reporting value. For AA and ICP analyses, a "less than" symbol (<) entered in the tables indicates that an element was below the lowest reporting value. If an element was above the highest reporting value, a "greater than" symbol (>) was entered in the tables. Because of the formatting used in the computer program that produced tables 4-6, some of the elements 1isted in these tables ( $\mathrm{Ca}, \mathrm{Fe}, \mathrm{Mg}, \mathrm{P}, \mathrm{Ti}$, and $\mathrm{Be}$ ) carry one or more nonsignificant digits to the right of the significant digits.

Some elements were not detected in any sample by emission spectrography and are omitted from tables 4-5. These elements are As, $\mathrm{Au}, \mathrm{Bi}, \mathrm{Cd}, \mathrm{Mo}, \mathrm{Sb}$, $\mathrm{Th}$, and $W$ in stream-sediment samples and $\mathrm{As}, \mathrm{Au}, \mathrm{Cd}, \mathrm{Ge}, \mathrm{Sb}, \mathrm{Pd}$, and $\mathrm{Pt}$ in nonmagnetic heavy-mineral-concentrate samples. Concentrations of $\mathrm{Bi}$ and $\mathrm{Sb}$, as determined by ICP, are all less than the lower limits of determination and thus are omitted from table 4. 


\section{REFERENCES CITED}

Bohannon, R.G., compiler, 1978, Preliminary geologic map of Las Vegas $1^{\circ} \times 2^{\circ}$ quadrangle, Nevada, Arizona and Cal ifornia: U.S. Geological Survey OpenFile Report 78-670, 6 p., scale 1:250,000.

1979 , Strike-slip faults of the Lake Mead region of southern Nevada, in Armentrout, J.M., Cole, M.R., and TerBest, Harry, Jr., eds., Cenozoic paleogeography of the western United States: Pacific Coast

Paleogeography Symposium 3, Pacific Section, Society of Economic

Paleontologists and Mineralogists, Los Angeles, Cal ifornia, p. 129-139.

Causey, J.D., 1988, Mineral resources of the Million Hills Wilderness Study Area, Clark County, Nevada: U.S. Bureau of Mines Mineral Land Assessment Open File Report MLA 34-88, 29 p.

Centanni, F.A., Ross, A.M., and DeSesa, M.A., 1956, Fluorometric determination of uranium: Analytical Chemistry, v. 28, p. 1651.

Crock, J. G., Briggs, P. H., Jackson, L.L., and Lichte, F.E., 1987, Analytical methods for the analysis of stream sediments and rocks from wilderness study areas: U.S. Geological Survey Open-File Report 87-84, 35 p.

Grimes, D. J., and Marranzino, A. P., 1968, Direct-current arc and alternating-current spark emission spectrographic field methods for the semiquantitative analys is of geologic materials: U.S. Geological Survey Circular 591, $6 \mathrm{p}$.

Longwe 11, C.R., Pampeyan, E.H., Bowyer, Ben, and Roberts, R.J., 1965, Geology and mineral deposits of Clark County, Nevada: Nevada Bureau of Mines and Geology Bulletin 62, 218 p., 16 plates.

Meier, A.L., 1980, Flameless atomic-absorption determination of gold in geological materials: Journal of Geochemical Exploration, v. 13, p. 77-85.

Motooka, J. M., and Grimes, D. J., 1976, Analytical precision of one-sixth order semiquantitative spectrographic analyses: U.S. Geological Survey Circular 738, $25 \mathrm{p}$.

O'Leary, R.M., and Meier, A.L., 1986, Analytical methods used in geochemical exploration, 1984: U.S. Geological Survey Circular 948, 48 p.

Stewart, J.H., and Carlson, J.E., compilers, 1978, Geologic map of Nevada: U.S. Geological Survey, scale 1:500,000.

Thompson, C. E., Nakagawa, H. M., and Van Sickle, G. H., 1968, Rapid analysis for gold in geologic materials, in Geological Survey Research 1968: U.S. Geological Survey Professional Paper 600-B, p. B130-B132.

VanTrump, George, Jr., and Miesch, A. T., 1977, The U.S. Geological Survey RASS-STATPAC system for management and statistical reduction of geochemical data: Computers and Geosciences, v. 3, p. 475-488.

Winters, R.A., 1988, Mineral resources of the Lime Canyon Wilderness Study Area, Clark County, Nevada: U.S. Bureau of Mines Mineral Land Assessment Open File Report MLA 40-88, 42 p. 
TABLE 1.--Limits of determination for the spectrographic analysis of stream sediments, based on a 10 -mg sample

\begin{tabular}{|c|c|c|}
\hline Elements & Lower determination limit & Upper determination $1 \mathrm{imit}$ \\
\hline \multicolumn{3}{|c|}{ Weight percent } \\
\hline $\begin{array}{l}\text { Calcium (Ca) } \\
\text { Iron (Fe) } \\
\text { Magnesium (Mg) } \\
\text { Titanium ( } \mathrm{Ti})\end{array}$ & $\begin{array}{l}0.05 \\
0.05 \\
0.02 \\
0.002\end{array}$ & $\begin{array}{r}20 \\
20 \\
10 \\
1\end{array}$ \\
\hline \multicolumn{3}{|c|}{ Parts per million } \\
\hline $\begin{array}{l}\text { Silver (Ag) } \\
\text { Arsenic (As) } \\
\text { Gold (Au) } \\
\text { Boron (B) } \\
\text { Barium (Ba) } \\
\text { Beryllium (Be) } \\
\text { Bismuth (Bi) } \\
\text { Cadmium (Cd) } \\
\text { Cobalt (Co) } \\
\text { Chromium (Cr) } \\
\text { Copper (Cu) } \\
\text { Lanthanum (La) } \\
\text { Manganese (Mn) } \\
\text { Molybdenum (Mo) } \\
\text { Niobium (Nb) } \\
\text { Nickel (Ni) } \\
\text { Lead (Pb) } \\
\text { Antimony (Sb) } \\
\text { Scandium (Sc) } \\
\text { Tin (Sn) } \\
\text { Strontium (Sr) } \\
\text { Thorium (Th) } \\
\text { Vanadium (V) } \\
\text { Tungsten (W) } \\
\text { Yttrium (Y) } \\
\text { Zinc ( } \mathrm{Zn} \text { ) } \\
\text { Zirconium (Zr) }\end{array}$ & $\begin{array}{r}0.5 \\
200 \\
10 \\
10 \\
20 \\
1 \\
10 \\
20 \\
5 \\
10 \\
5 \\
20 \\
10 \\
5 \\
20 \\
5 \\
10 \\
100 \\
5 \\
10 \\
100 \\
100 \\
10 \\
50 \\
10 \\
200 \\
10\end{array}$ & $\begin{array}{r}5,000 \\
10,000 \\
500 \\
2,000 \\
5,000 \\
1,000 \\
1,000 \\
500 \\
2,000 \\
5,000 \\
20,000 \\
1,000 \\
5,000 \\
2,000 \\
2,000 \\
5,000 \\
20,000 \\
10,000 \\
100 \\
1,000 \\
5,000 \\
2,000 \\
10,000 \\
10,000 \\
2,000 \\
10,000 \\
1,000\end{array}$ \\
\hline
\end{tabular}


TABLE 2.--Limits of determination for the spectrographic analysis of normagnetic heavy-mineral-concentrate samples, based on a 5-mg sample

\begin{tabular}{|c|c|c|}
\hline Elements & Lower determination limit & Upper determination limit \\
\hline \multicolumn{3}{|c|}{ Percent } \\
\hline $\begin{array}{l}\text { Calcium (Ca) } \\
\text { Iron ( } \mathrm{Fe}) \\
\text { Magnesium (Mg) } \\
\text { Sodium (Na) } \\
\text { Phosphorus (P) } \\
\text { Titanium (Ti) }\end{array}$ & $\begin{array}{l}0.1 \\
0.1 \\
0.05 \\
0.5 \\
0.5 \\
0.005\end{array}$ & $\begin{array}{r}50 \\
50 \\
20 \\
10 \\
20 \\
2\end{array}$ \\
\hline \multicolumn{3}{|c|}{ Parts per million } \\
\hline $\begin{array}{l}\text { Silver (Ag) } \\
\text { Arsenic (As) } \\
\text { Gold (Au) } \\
\text { Boron (B) } \\
\text { Barium (Ba) } \\
\text { Beryllium (Be) } \\
\text { Bismuth (Bi) } \\
\text { Cadmium (Cd) } \\
\text { Cobalt (Co) } \\
\text { Chromium (Cr) } \\
\text { Copper (Cu) } \\
\text { Gallium (Ga) } \\
\text { Germanium (Ge) } \\
\text { Lanthanum (La) } \\
\text { Manganese (Mn) } \\
\text { Molybdenum (Mo) } \\
\text { Niobium (Nb) } \\
\text { Nickel (Ni) } \\
\text { Lead (Pb) } \\
\text { Antimony (Sb) } \\
\text { Scandium (Sc) } \\
\text { Tin (Sn) } \\
\text { Strontium (Sr) } \\
\text { Thorium (Th) } \\
\text { Vanadium (V) } \\
\text { Tungsten (W) } \\
\text { Yttrium (Y) } \\
\text { Zinc ( } \mathrm{Zn} \text { ) } \\
\text { Zirconium ( }(\mathrm{Ir} \text { ) } \\
\text { Palladium (Pd) } \\
\text { Platinum (Pt) }\end{array}$ & $\begin{array}{r}1 \\
500 \\
20 \\
20 \\
50 \\
2 \\
20 \\
50 \\
20 \\
20 \\
10 \\
10 \\
20 \\
100 \\
20 \\
10 \\
50 \\
10 \\
20 \\
200 \\
10 \\
20 \\
200 \\
200 \\
20 \\
50 \\
20 \\
500 \\
20 \\
5 \\
20\end{array}$ & $\begin{array}{r}10,000 \\
20,000 \\
1,000 \\
5,000 \\
10,000 \\
2,000 \\
2,000 \\
1,000 \\
5,000 \\
10,000 \\
50,000 \\
1,000 \\
200 \\
2,000 \\
10,000 \\
5,000 \\
5,000 \\
10,000 \\
50,000 \\
20,000 \\
200 \\
2,000 \\
10,000 \\
5,000 \\
20,000 \\
20,000 \\
5,000 \\
20,000 \\
2,000 \\
1,000 \\
1,000\end{array}$ \\
\hline
\end{tabular}


TABLE 3.--Analytical methods used other than emission spectrography

[AAF, flame atomic absorption; AAG, graphite furnace atomic absorption; $F$, ultraviolet fluorimetry; ICP, inductively coupled plasma-atomic emission spectroscopy]

\begin{tabular}{|c|c|c|c|c|c|}
\hline $\begin{array}{l}\text { Element } \\
\text { determined }\end{array}$ & $\begin{array}{l}\text { Sample } \\
\text { type }\end{array}$ & Method & $\begin{array}{l}\text { Lower } \\
\text { determin- } \\
\text { ation limit, } \\
\text { ppm }\end{array}$ & $\begin{array}{l}\text { Precision, } \\
\text { percent } \\
\text { relative } \\
\text { standard } \\
\text { deviation }\end{array}$ & References \\
\hline $\begin{array}{l}\text { Arsenic (As) } \\
\text { Antimony (Sb) } \\
\text { Bismuth (Bi) } \\
\text { Cadmium (Cd) } \\
\text { Zinc ( } \mathrm{Zn})\end{array}$ & $\begin{array}{l}\text { stream sediment } \\
\text { stream sediment } \\
\text { stream sediment } \\
\text { stream sediment } \\
\text { stream sediment }\end{array}$ & $\begin{array}{l}\text { ICP } \\
\text { ICP } \\
\text { ICP } \\
\text { ICP } \\
\text { ICP }\end{array}$ & $\begin{array}{l}5 \\
2 \\
2 \\
0.1 \\
2\end{array}$ & $\begin{array}{l}3.5-20 \\
6.4-11 \\
2.2-11.9 \\
2.8-8.8 \\
1.4-11.9\end{array}$ & $\begin{array}{l}\text { Crock and } \\
\text { others, } 1987 .\end{array}$ \\
\hline Uranium (U) & stream sediment & $F$ & 0.1 & $6.9-14.2$ & $\begin{array}{l}\text { Centanni and } \\
\text { others, 1956; } \\
\text { o'Leary and } \\
\text { Meier, } 1986 .\end{array}$ \\
\hline Gold $(\mathrm{Au})$ & stream sediment & AAG & 0.001 & $3.7-21.1$ & $\begin{array}{l}\text { Meier, 1980; } \\
\text { O'Leary and } \\
\text { Me ier, } 1986 .\end{array}$ \\
\hline Gold $(A u)$ & $\begin{array}{l}\text { raw panned } \\
\text { concentrate }\end{array}$ & AAF & $0.05^{\star}$ & $9.3-42.5$ & $\begin{array}{l}\text { Thompson and } \\
\text { others, 1968; } \\
\text { O'Leary and } \\
\text { Meier, } 1986 .\end{array}$ \\
\hline
\end{tabular}

$-$

${ }^{\star}$ Based on $10-g$ sample 
TABLE 4.--Results of analyses of streau-sediuent samples from the Liwe Canyon and Million Hills Hilderness Study Areas. Clark County. Nevada.

[N, not detected: (. detected beiow concentfation shown for euission spectrodraphic analyses. less than concentration shown for other methods; areater than concentration showri. Methods: Au-a, graphite furnace atouic absorption spectroscopy: His-i. Cd-i. Zn-i, inductively coupled plasta-atouic euission spectroscopy; U-f, ultraviolet fluoriuetry: others, euission spectrogaphy. Concentrations in ppw except $\mathrm{Ca}, \mathrm{Fe}$, Mgg, and $\mathrm{Ti}$, which are weight percent]

\begin{tabular}{|c|c|c|c|c|c|c|c|c|c|c|c|c|c|c|}
\hline Sample & Latitude & Longitude & $\mathrm{Ca}$ & $\mathrm{Fe}$ & $\mathrm{Mg}_{L_{1}}$ & $\begin{array}{c}\mathrm{Ti} \\
\text { Canyon }\end{array}$ & $A Q$ & $B$ & $\mathrm{Ba}$ & Be & $C_{0}$ & $\mathrm{Cr}$ & Cu & $L$ \\
\hline LIMO33 & $36 \quad 1558$ & $11416 \quad 8$ & 2.0 & 3.00 & 3.0 & .70 & N & 30 & 500 & 1.5 & 30 & 100 & 50 & \\
\hline LIM034 & 36164 & 1141634 & .7 & 5.00 & 1.5 & 1.00 & $N$ & 70 & 700 & 2.0 & 50 & 50 & 50 & 100 \\
\hline LIN035 & 361625 & 114177 & 1.5 & 3.00 & 5.0 & .70 & N & 50 & 700 & 2.0 & 20 & 100 & 70 & 70 \\
\hline LIM036 & $36 \quad 1648$ & 1141839 & 2.0 & 5.00 & 2.0 & 1.00 & $N$ & 50 & 700 & 1.5 & 20 & 70 & 50 & 150 \\
\hline LIM037 & 361725 & 114208 & 1.5 & 5.00 & 2.0 & 1.00 & $N$ & 50 & 500 & 1.5 & 20 & 70 & 50 & 100 \\
\hline LIH038 & 361728 & 114207 & 1.5 & 5.00 & 1.5 & 1.00 & $N$ & 50 & 500 & 2.0 & 20 & 70 & 30 & 10 \\
\hline LIn039 & $\begin{array}{lll}36 & 18 & 0\end{array}$ & 1141936 & 2.0 & 3.00 & 2.0 & .70 & N & 50 & 700 & 1.5 & 30 & 70 & 30 & 10 \\
\hline LIM040 & 361811 & 1141844 & 2.0 & 3.00 & 5.0 & .50 & $N$ & 30 & 700 & 2.0 & 15 & 50 & 30 & 70 \\
\hline LIM041 & 361711 & 1141923 & 1.5 & 3.00 & 1.5 & .50 & $N$ & 30 & 700 & 2.0 & 15 & 70 & 30 & 100 \\
\hline LInO42 & 361650 & 1142027 & 1.0 & 3.00 & 1.0 & .70 & $N$ & 10 & 500 & 1.5 & 15 & 50 & 20 & 70 \\
\hline LIMO43 & 361623 & 114200 & 1.5 & 3.00 & 1.0 & .70 & N & 20 & 700 & 2.0 & 20 & 50 & 20 & 70 \\
\hline LIM101 & 361754 & $114 \quad 13 \quad 13$ & 1.5 & 3.00 & 1.5 & .50 & N & 10 & 300 & 3.0 & 20 & 50 & 20 & 30 \\
\hline LIMLO2 & 361824 & $11414 \quad 8$ & 3.0 & 5.00 & 5.0 & .30 & N & 20 & 500 & 3.0 & 10 & 20 & 20 & 70 \\
\hline LIM103 & 361825 & $11414 \quad 13$ & 2.0 & 1.00 & 1.0 & .20 & N & 30 & 300 & $N$ & $N$ & 30 & 10 & 20 \\
\hline LIMIO4 & 362051 & 1141412 & 2.0 & 2.00 & 3.0 & .20 & $N$ & 70 & 300 & 1.0 & 15 & 20 & 30 & $<20$ \\
\hline LIMI05 & 362050 & 1141416 & 2.0 & 1.00 & 1.5 & .20 & $N$ & 50 & 500 & $\langle 1.0$ & 5 & 10 & 10 & 20 \\
\hline LIM106 & 362130 & 1141433 & 1.5 & .70 & 1.0 & .15 & $N$ & 50 & 300 & $<1.0$ & $<5$ & 15 & 20 & \\
\hline LIMlo7 & 362153 & 1141418 & 2.0 & .70 & 1.0 & .15 & N & 50 & 300 & N & $<5$ & 20 & 15 & $<20$ \\
\hline LIM108 & $\begin{array}{lll}36 & 22 & 20\end{array}$ & 1141424 & 2.0 & 1.00 & .7 & .20 & $N$ & 30 & 300 & $N$ & 5 & 15 & 10 & $<20$ \\
\hline LIMI09 & 36237 & 114144 & 2.0 & 1.50 & 1.0 & .30 & $N$ & 30 & 700 & $<1.0$ & 10 & 30 & 20 & 30 \\
\hline LIn110 & 362350 & 1141353 & 2.0 & 1.50 & 1.0 & .30 & $N$ & 50 & 500 & 1.0 & 5 & 30 & 15 & 30 \\
\hline LIn11I & 362413 & 1141344 & 2.0 & 2.00 & .7 & .30 & N & 20 & 500 & 1.5 & 5 & 30 & 15 & 20 \\
\hline LInI12 & 362450 & 1141356 & 3.0 & 2.00 & 2.0 & .20 & $N$ & 30 & 300 & $<1.0$ & 7 & $<10$ & 20 & $<20$ \\
\hline LIMI13 & 362554 & 1141346 & 2.0 & 1.50 & 1.0 & .20 & $N$ & 50 & 500 & $<1.0$ & $<5$ & 10 & 10 & 20 \\
\hline LIMI14 & 362645 & 1141415 & 1.5 & 2.00 & 1.5 & .15 & N & 50 & 1.000 & 1.5 & 10 & 20 & 30 & 20 \\
\hline LIM115 & 362653 & 114161 & 2.0 & 3.00 & 2.0 & .50 & N & 70 & 700 & 1.5 & 15 & 20 & 30 & 30 \\
\hline LIM116 & 362628 & 1141650 & 3.0 & 2.00 & 5.0 & .15 & N & 100 & 500 & 1.5 & 5 & 10 & 30 & 20 \\
\hline LIM117 & $\begin{array}{lll}36 & 26 & 3\end{array}$ & 1141639 & 1.0 & .50 & .7 & .10 & N & 50 & 1.000 & N & $N$ & N & 5 & $N$ \\
\hline LIM118 & $36 \quad 2530$ & 1141624 & 2.0 & .70 & 1.0 & .10 & $N$ & 50 & 700 & $<1.0$ & $N$ & 50 & 7 & N \\
\hline LIM119 & $3625 \quad 2$ & 1141622 & 1.5 & .30 & 1.0 & .07 & $N$ & 30 & 500 & N & $N$ & N & $<5$ & N \\
\hline LIM120 & 362427 & 1141719 & 2.0 & 2.00 & 2.0 & .70 & N & 50 & 500 & $<1.0$ & 5 & 20 & 20 & 30 \\
\hline LIM121 & $\begin{array}{lll}36 & 23 & 7\end{array}$ & 1141726 & 3.0 & 1.50 & 3.0 & .20 & N & 30 & 500 & $<1.0$ & 5 & 10 & 15 & 20 \\
\hline LIM122 & 362238 & 114175 & 2.0 & 1.00 & 5.0 & .10 & $N$ & 30 & 300 & N & $<5$ & $<10$ & 10 & N \\
\hline LIMI23 & 36226 & 1141820 & 2.0 & 2.00 & 3.0 & .20 & $N$ & 50 & 1.500 & 1.0 & 15 & 10 & 30 & 20 \\
\hline LIM124 & 362210 & 1141745 & 2.0 & .50 & 2.0 & .10 & $N$ & 50 & 300 & $N$ & $N$ & 10 & 5 & $<20$ \\
\hline LIM125 & 362233 & $11418 \quad 3$ & 3.0 & .70 & 5.0 & .10 & $N$ & 20 & 200 & $N$ & $<5$ & 10 & 10 & 20 \\
\hline LIM126 & 362141 & $114 \quad 1817$ & 1.0 & .50 & .7 & .10 & $N$ & 30 & 500 & N & $N$ & $<10$ & $<5$ & $A$ \\
\hline LIM127 & $\begin{array}{llll}36 & 21 & 47\end{array}$ & $114 \quad 1847$ & 2.0 & 3.00 & 3.0 & .50 & $N$ & 50 & 500 & $<1.0$ & 10 & 30 & 20 & 20 \\
\hline LIM128 & 362039 & 1141911 & 3.0 & 2.00 & 5.0 & .20 & N & 70 & 300 & $<1.0$ & $<5$ & 20 & 7 & 30 \\
\hline LIM129 & 361938 & $114 \quad 1831$ & 2.0 & 1.00 & 2.0 & .20 & $N$ & 70 & 700 & $<1.0$ & $<5$ & 30 & 10 & 20 \\
\hline
\end{tabular}


TABLE 4,--Results of analyses of stream-sediment samples from the Lime Canyon and Million Hills Hilderness Study Areas, Clark County, Nevada--Contanued

\begin{tabular}{|c|c|c|c|c|c|c|c|c|c|c|c|c|c|c|c|c|}
\hline Sauple & $\mathrm{Mn}$ & $\mathrm{Nb}$ & $\mathrm{Ni}$ & $\mathrm{Pb}$ & $\mathrm{Sc}$ & Sn & $\begin{array}{c}\text { Sr } \\
\text { Lime C }\end{array}$ & $\begin{array}{c}U \\
\text { yon }--\end{array}$ & $\begin{array}{c}\text { Y } \\
\text { ntinued }\end{array}$ & $2 n$. & $2 r$ & $A_{5}-i$ & $C d-i$ & $2 n-i$ & $A u-a$ & $U-f$ \\
\hline LIM033 & 1.000 & $<20$ & 200 & 20 & 20 & $<10$ & 150 & 100 & 50 & $<200$ & 300 & 6 & 1.5 & 60 & .001 & 1.30 \\
\hline LIM034 & 700 & $<20$ & 50 & 50 & 20 & 10 & $<100$ & 100 & 70 & $<200$ & 500 & 16 & 1.7 & 130 & .001 & 1.10 \\
\hline LIM035 & 1,500 & N & 50 & 200 & 20 & $<10$ & 200 & 150 & 50 & 200 & 200 & 12 & 2.3 & 270 & .006 & 1.20 \\
\hline LIM036 & 1,000 & $<20$ & 50 & 30 & 20 & $<10$ & 200 & 150 & 100 & $N$ & 700 & 8 & 1.4 & 64 & .001 & 1.90 \\
\hline LIM037 & 1,000 & $<20$ & 50 & 30 & 20 & $<10$ & 100 & 150 & 100 & $N$ & 1.000 & $<5$ & 1.2 & 60 & .001 & 1.90 \\
\hline LIM038 & 1,000 & $<20$ & 30 & 20 & 20 & $<10$ & 100 & 150 & 70 & $N$ & 1,000 & $<5$ & 1.3 & 59 & .001 & 1.50 \\
\hline LIM039 & 700 & $<20$ & 30 & 20 & 20 & $N$ & 200 & 150 & 70 & $N$ & 1,000 & 8 & .9 & 52 & .001 & 1.10 \\
\hline LIM040 & 500 & $N$ & 30 & 30 & 15 & $N$ & 200 & 100 & 50 & $<200$ & 300 & 8 & .9 & 71 & .001 & .90 \\
\hline LIMO4I & 500 & $<20$ & 30 & 20 & 20 & N & 200 & 100 & 70 & $<200$ & 300 & 7 & 1.2 & 59 & .001 & 1.90 \\
\hline LIM042 & 500 & $<20$ & 20 & 20 & 20 & $N$ & 100 & 70 & 50 & $N$ & 700 & 7 & 1.2 & 54 & .001 & 1.80 \\
\hline LIMO43 & 700 & $<20$ & 30 & 20 & 20 & N & 200 & 100 & 50 & $<200$ & 300 & 7 & 1.6 & 68 & .001 & 1.30 \\
\hline LIM101 & 500 & $<20$ & 50 & 20 & 15 & $N$ & 200 & 70 & 30 & $N$ & 500 & $<5$ & 1.2 & 45 & .001 & 1.20 \\
\hline LIM102 & 1.000 & $<20$ & 20 & 20 & 15 & $<10$ & 150 & 100 & 30 & $<200$ & 300 & 6 & 2.6 & 44 & .001 & 1.30 \\
\hline LIMIO3 & 300 & $N$ & 5 & 20 & 5 & $N$ & 150 & 50 & 10 & $N$ & 150 & 8 & .9 & 26 & .001 & 1.90 \\
\hline LIM104 & 500 & $N$ & 20 & 15 & 7 & $\mathbf{N}$ & 500 & 70 & 10 & $N$ & 200 & 7 & 1.2 & 31 & .001 & 1.10 \\
\hline LIM105 & 200 & $\mathbf{N}$ & 10 & 20 & 5 & $N$ & 100 & 30 & 15 & $N$ & 500 & 7 & .5 & 24 & $<.001$ & .75 \\
\hline LIM106 & 150 & $N$ & 7 & 20 & $N$ & $N$ & $<100$ & 20 & $<10$ & $N$ & 100 & 8 & .5 & 29 & .001 & 1.00 \\
\hline LIM107 & 150 & $N$ & 5 & 20 & $N$ & $N$ & 100 & 20 & 10 & $N$ & 100 & 11 & .5 & 22 & .001 & 1.50 \\
\hline LIMIOB & 200 & $N$ & 7 & 15 & $N$ & N & 150 & 30 & 15 & $N$ & 200 & 5 & .6 & 26 & .001 & .55 \\
\hline LIM109 & 200 & $<20$ & 20 & 30 & 7 & $N$ & 150 & 50 & 20 & $N$ & 300 & 8 & .6 & $4 !$ & .001 & .75 \\
\hline LIMIIO & 300 & $<20$ & 15 & 30 & 7 & $N$ & 200 & 50 & 20 & $N$ & 300 & 14 & .7 & 43 & .001 & .85 \\
\hline LIM111 & 300 & $<20$ & 10 & 20 & 5 & $N$ & 100 & 50 & 20 & $N$ & 500 & 8 & .8 & 41 & .001 & .75 \\
\hline LIM112 & 200 & $<20$ & 10 & 20 & 7 & $N$ & 500 & 50 & 15 & $N$ & 200 & 8 & .6 & 30 & $<.001$ & .90 \\
\hline LIn113 & 300 & $N$ & 5 & 30 & 5 & $N$ & 150 & 50 & 15 & $N$ & 200 & 6 & .6 & 32 & .001 & 1.20 \\
\hline LIM114 & 500 & $N$ & 10 & 10 & 7 & $N$ & 200 & 50 & 10 & $N$ & 70 & $<5$ & .8 & 50 & .002 & 1.30 \\
\hline LIM115 & 700 & $<20$ & 20 & 20 & 10 & N & 200 & 100 & 20 & $<200$ & 200 & 6 & .9 & 43 & .002 & 1.40 \\
\hline LIM116 & 500 & $N$ & 15 & 20 & 7 & $N$ & 1.500 & 50 & 10 & $<200$ & 70 & 6 & .6 & 44 & .002 & 1.20 \\
\hline LIML17 & 100 & $N$ & 5 & $N$ & $N$ & $N$ & 150 & 10 & N & $N$ & 200 & $<5$ & .2 & 7 & .002 & .30 \\
\hline LIM118 & 100 & $N$ & 5 & $N$ & $N$ & $N$ & 100 & 20 & $<10$ & $N$ & 300 & 6 & .3 & 11 & $<.001$ & .55 \\
\hline LIMI19 & 70 & $N$ & 5 & N & $N$ & N & $\langle 100$ & 10 & $N$ & $N$ & 200 & 6 & .2 & 6 & $<.001$ & .45 \\
\hline LIM120 & 500 & $<20$ & 10 & 15 & 7 & $N$ & 100 & 50 & 20 & $N$ & 700 & 12 & .9 & 27 & $<.001$ & 1.40 \\
\hline LIM121 & 300 & $N$ & 10 & 15 & 5 & $N$ & 100 & 50 & 15 & $N$ & 100 & 9 & .7 & 26 & .001 & 1.00 \\
\hline LIM122 & 200 & $N$ & 5 & 20 & $<5$ & $N$ & $<100$ & 20 & $<10$ & $N$ & 100 & 9 & .5 & 17 & .001 & .80 \\
\hline LIM123 & 500 & $N$ & 10 & 10 & 7 & $N$ & 1.000 & 50 & 15 & $<200$ & 150 & $<5$ & .9 & 33 & .001 & 1.90 \\
\hline LIMI24 & 200 & $N$ & $<5$ & $N$ & $<5$ & $N$ & 100 & 20 & N & $N$ & 300 & $<5$ & .2 & 7 & $<.001$ & .80 \\
\hline LIM125 & 200 & $N$ & 5 & 10 & $<5$ & $N$ & 100 & 30 & 20 & $N$ & 200 & 6 & .4 & 11 & $<.001$ & .80 \\
\hline LIM126 & 100 & $\mathrm{~N}$ & 5 & $<10$ & $\mathrm{~N}$ & N & $\langle 100$ & 15 & 10 & $N$ & 200 & $<5$ & .2 & ó & .006 & .40 \\
\hline LIM127 & 500 & $<20$ & 15 & 20 & 10 & $N$ & 100 & 100 & $<10$ & $\mathrm{~N}$ & 500 & 10 & 1.1 & 20 & $<.001$ & 1.10 \\
\hline LIM128 & 700 & $N$ & 7 & 20 & 5 & $N$ & 100 & 50 & N & $\langle 200$ & 150 & $<5$ & 1.6 & 26 & .001 & .80 \\
\hline LIMI29 & 300 & $N$ & 10 & 10 & 5 & $N$ & 150 & 30 & 15 & $N$ & 500 & 9 & .4 & 13 & $<.001$ & .50 \\
\hline
\end{tabular}


TABLE 4.--Results of analyses of strean-sediment samples from the Lime Canyon and Million Hills Hilderness Study Areas, Clark County, Nevada--Continued

\begin{tabular}{|c|c|c|c|c|c|c|c|c|c|c|c|c|c|c|}
\hline Sauple & Latitude & Longitude & $\mathrm{Ca}$ & $\mathrm{Fe}$ & $\stackrel{M g}{\mathrm{M}}$ & $\begin{array}{c}T i \\
n--\operatorname{Cont}\end{array}$ & Aq & -8 & $\mathrm{Ba}$ & $\mathrm{Be}$ & Co & $\mathrm{Cr}$ & $\mathrm{Cu}$ & La \\
\hline LIMI30 & $36 \quad 1926$ & 1141743 & 1.5 & 2.00 & 3.0 & .50 & $N$ & 100 & 700 & 1.0 & 10 & 20 & 20 & 20 \\
\hline LIM131 & 361913 & $114 \quad 18 \quad 21$ & 2.0 & 7.00 & 3.0 & .70 & $\lfloor .5$ & 50 & 700 & 1.5 & 20 & 200 & 50 & 70 \\
\hline LIM132 & 361855 & 1141823 & 2.0 & 5.00 & 2.0 & .50 & $N$ & 30 & 500 & 1.0 & 20 & 30 & 30 & 20 \\
\hline LIM133 & $36 \quad 1841$ & 1141836 & 2.0 & 5.00 & 5.0 & .50 & $N$ & 100 & 500 & 2.0 & 30 & 50 & 50 & 50 \\
\hline LIMI34 & 361613 & $114 \quad 1527$ & 2.0 & 5.00 & 3.0 & .50 & $N$ & 10 & 500 & $<1.0$ & 30 & 70 & 50 & $<20$ \\
\hline LIM135 & 361618 & 1141521 & 2.0 & 2.00 & 2.0 & .50 & $N$ & 30 & 300 & 1.0 & 20 & 50 & 30 & 30 \\
\hline LIN136 & $36 \quad 1557$ & 114151 & 1.5 & 5.00 & 2.0 & .70 & $N$ & 20 & 300 & 1.0 & 50 & 100 & 50 & 30 \\
\hline LIM137 & 361551 & 1141415 & 1.0 & 3.00 & 1.5 & .50 & $N$ & 15 & 300 & 1.5 & 20 & 30 & 30 & 20 \\
\hline LIM138 & 361543 & 1142037 & 2.0 & 5.00 & 1.0 & $>1.00$ & $N$ & 30 & 700 & 2.0 & 20 & 70 & 50 & 100 \\
\hline LIN139 & $36 \quad 18 \quad 47$ & $114 \quad 1434$ & 1.5 & 3.00 & 1.0 & .70 & $N$ & 20 & 300 & $<1.0$ & 7 & 100 & 15 & 50 \\
\hline LIM140 & 361840 & 1141436 & 3.0 & .50 & 1.5 & .20 & $N$ & 30 & 300 & N & N & 10 & $<5$ & N \\
\hline LIM141 & $3619 \quad 14$ & 1141556 & 10.0 & .70 & 10.0 & .07 & $N$ & 30 & 300 & $N$ & $<5$ & $N$ & 10 & N \\
\hline LIM142 & $36 \quad 1922$ & 1141623 & 1.5 & 3.00 & 1.5 & .70 & $N$ & 50 & 150 & 2.0 & 15 & 100 & 20 & 50 \\
\hline LIII43 & 361925 & 1141616 & 7.0 & 1.00 & 5.0 & .10 & $N$ & 30 & 500 & $N$ & $<5$ & 10 & 10 & $<20$ \\
\hline LIMI44 & $\begin{array}{lll}36 & 19 & 0\end{array}$ & 1141531 & 7.0 & .07 & 5.0 & .05 & $N$ & 30 & 300 & $N$ & $N$ & N & 10 & $<20$ \\
\hline LIM145 & $3619 \quad 1$ & $114 \quad 1545$ & 7.0 & 2.00 & 5.0 & .15 & $N$ & 70 & 150 & 1.5 & $<5$ & $<10$ & 10 & $N$ \\
\hline LIMI46 & 361952 & 1141659 & 7.0 & 2.00 & 3.0 & .20 & $N$ & 30 & 200 & $N$ & 5 & $<10$ & 15 & 20 \\
\hline LIMI47 & 361951 & 1141653 & 5.0 & 3.00 & 2.0 & .30 & $N$ & 50 & 300 & 1.0 & 5 & 10 & 15 & 50 \\
\hline LIMI48 & 362331 & 114157 & 3.0 & 1.00 & .7 & .10 & $N$ & 50 & 500 & N & N & 20 & 7 & N \\
\hline LIM149 & 362335 & $114 \quad 15 \quad 5$ & 1.5 & .70 & .5 & .07 & $N$ & 50 & 500 & N & $N$ & 10 & 5 & N \\
\hline
\end{tabular}

\section{Million Hills}

\begin{tabular}{|c|c|c|c|c|c|c|c|c|c|c|c|c|c|c|}
\hline HILO44 & 361910 & 114 & 520 & 7.0 & .50 & 10.0 & .05 & $N$ & 15 & 100 & $N$ & N & N & 7 \\
\hline MILO45 & 361910 & 114 & 521 & 2.0 & 3.00 & 5.0 & .20 & $N$ & 30 & 300 & 3.0 & 15 & 20 & 20 \\
\hline MILI50 & 361852 & 114 & 330 & 1.5 & 3.00 & 2.0 & .50 & $N$ & 10 & 500 & 3.0 & 20 & 100 & 30 \\
\hline HIL151 & 361935 & 114 & 258 & 3.0 & 10.00 & 1.5 & 1.00 & $N$ & 30 & 300 & 3.0 & 50 & 500 & 50 \\
\hline HILI52 & 361858 & 114 & 222 & 2.0 & 5.00 & 2.0 & .50 & $N$ & 20 & 300 & $<1.0$ & 20 & 200 & 50 \\
\hline MILI53 & $36 \quad 2031$ & 114 & 250 & 3.0 & 1.50 & 2.0 & .15 & $N$ & 30 & 500 & 1.5 & 10 & 50 & 20 \\
\hline MIL154 & 362033 & 114 & 247 & 2.0 & 2.00 & 2.0 & .20 & $N$ & 20 & 300 & 1.0 & 15 & 70 & 20 \\
\hline MIL155 & 361947 & 114 & 232 & 2.0 & 3.00 & 3.0 & .20 & $N$ & 50 & 500 & 3.0 & 20 & 150 & 20 \\
\hline MIL156 & 361955 & 114 & 235 & 1.5 & 5.00 & 3.0 & .30 & $N$ & 30 & 300 & 2.0 & 30 & 200 & 30 \\
\hline MILI57 & 361736 & 114 & 234 & 2.0 & 1.50 & 7.0 & .15 & $N$ & 30 & 200 & $\langle 1.0$ & 7 & 1.000 & 15 \\
\hline MIL158 & 361741 & 114 & 231 & 3.0 & 1.00 & 5.0 & .15 & $N$ & 30 & 300 & N & 10 & $<10$ & 10 \\
\hline MILI59 & $36 \quad 16 \quad 12$ & 114 & 336 & 2.0 & 2.00 & 2.0 & .30 & $N$ & 70 & 500 & $N$ & 5 & 100 & 7 \\
\hline HILI60 & $36 \quad 16 \quad 14$ & 114 & 338 & 1.5 & .50 & 1.0 & .10 & $N$ & 50 & 300 & N & $<5$ & 20 & $<5$ \\
\hline MILI61 & 361616 & 114 & 336 & 3.0 & .50 & 3.0 & .07 & $N$ & 30 & 200 & $N$ & $<5$ & $<10$ & 5 \\
\hline MILI62 & $36 \quad 155$ & 114 & 58 & 1.0 & 5.00 & 2.0 & .50 & $N$ & 50 & 300 & 5.0 & 30 & 150 & 30 \\
\hline MILI63 & $\begin{array}{lll}36 & 15 & 3\end{array}$ & 114 & 57 & 1.5 & 5.00 & 3.0 & .70 & N & 20 & 500 & 3.0 & 50 & 200 & 50 \\
\hline MILI64 & 36155 & 114 & 25 & 5.0 & 2.00 & 2.0 & .30 & $\mathbf{N}$ & 50 & 500 & 2.0 & 10 & 70 & 20 \\
\hline MIL165 & $36 \quad 1539$ & 114 & 146 & 2.0 & 3.00 & 1.5 & .50 & $N$ & 20 & 500 & 3.0 & 20 & 150 & 20 \\
\hline HILI66 & $3617 \quad 0$ & 114 & 214 & 3.0 & 2.00 & 3.0 & .20 & $N$ & 50 & 300 & $<1.0$ & 10 & 20 & 30 \\
\hline MIL167 & 361551 & 114 & 21 & 2.0 & 2.00 & 1.0 & .50 & $N$ & 20 & 300 & 2.0 & 15 & 100 & 20 \\
\hline MIL168 & 36160 & 114 & 157 & 3.0 & 2.00 & 5.0 & .30 & $N$ & 20 & 300 & $N$ & 10 & 70 & 10 \\
\hline HILI69 & 361556 & 114 & 159 & 5.0 & 3.00 & 5.0 & .20 & $N$ & 20 & 500 & 1.0 & 10 & 100 & 15 \\
\hline NIL170 & 36143 & 114 & 312 & 2.0 & 5.00 & 5.0 & .50 & $N$ & 10 & 500 & 1.5 & 15 & 200 & 30 \\
\hline MILI71 & 361416 & 114 & 340 & 7.0 & 1.00 & 2.0 & .10 & $N$ & 30 & 200 & $N$ & 5 & 20 & 15 \\
\hline
\end{tabular}


TABLE 4.--Results of analyses of stream-sediment samples from the Lime Canyon and Million Hills Hilderness Study Areas. Clark County, Nevada--Continued

\begin{tabular}{|c|c|c|c|c|c|c|c|c|c|c|c|c|c|c|c|c|}
\hline Sauple & $M_{n}$ & $\mathrm{Nb}$ & $\mathrm{Ni}$ & $\mathrm{Pb}$ & Sc & $\mathrm{Sn}$ & $\mathrm{Sr}$ & $v$ & Y & $2 n-$ & $2 r$ & $A_{5}-\mathrm{i}$ & $C d-i$ & $2 n-i$ & $A u-a$ & $U-f$ \\
\hline & & & & & & 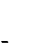 & Lime C & yon-- & ntint & & & & & & & \\
\hline LIK130 & 500 & $N$ & 15 & 20 & 10 & $N$ & 300 & 100 & 20 & $N$ & 200 & 8 & .8 & 25 & .002 & 2.10 \\
\hline LIM131 & 1.000 & $N$ & 70 & 30 & 20 & $<10$ & 500 & 300 & 30 & $<200$ & 200 & 26 & 2.8 & 53 & .002 & 1.10 \\
\hline LIn132 & 700 & $N$ & 50 & 30 & 15 & $N$ & 100 & 100 & 20 & $<200$ & 200 & 7 & 1.3 & 42 & .001 & 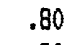 \\
\hline LIM133 & 700 & $<20$ & 70 & 30 & 20 & N & 100 & 150 & 30 & $<200$ & 500 & 7 & 1.3 & 48 & .003 & .9 \\
\hline LIM134 & 1.000 & $N$ & 150 & 10 & 15 & $N$ & 150 & 100 & 15 & $<200$ & 150 & 5 & 1.7 & 53 & .001 & .9 \\
\hline LIMI35 & 500 & N & 70 & 30 & 10 & N & 100 & 70 & 20 & $N$ & 200 & $\langle 5$ & .8 & 31 & $<.001$ & .80 \\
\hline LIM136 & 1,000 & $<20$ & 200 & 20 & 15 & N & 150 & 150 & 20 & $N$ & 500 & $<5$ & 2.3 & 63 & .001 & 3.30 \\
\hline LIM137 & 700 & $N$ & 70 & 30 & 15 & $N$ & $\$ 100$ & 100 & 30 & $N$ & 300 & $<5$ & 1.1 & 77 & .001 & 1.00 \\
\hline LIMI38 & 2,000 & $<20$ & 50 & 30 & 20 & $N$ & 200 & 200 & 70 & 200 & 500 & 10 & 2.7 & 88 & .001 & 1.5 \\
\hline LIM139 & 700 & $<20$ & 20 & 10 & 5 & $N$ & $\langle 100$ & 100 & 20 & $N$ & 700 & $<5$ & 1.6 & 32 & .001 & \\
\hline LIMI40 & 150 & $N$ & 5 & 10 & $N$ & $N$ & $\langle 100$ & 20 & $<10$ & $N$ & 300 & $<5$ & .1 & 7 & $<.001$ & 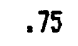 \\
\hline LIMI41 & 200 & $N$ & $<5$ & 20 & $N$ & $N$ & $\langle 100$ & 20 & 10 & $N$ & 100 & $<5$ & .4 & 6 & $<.001$ & .85 \\
\hline LIHI42 & 700 & $N$ & 20 & 15 & 10 & $N$ & 100 & 150 & 20 & $<200$ & 200 & 6 & 1.4 & 45 & .001 & 1.5 \\
\hline LIHI43 & 200 & $N$ & 10 & 20 & $<5$ & $N$ & 100 & 30 & $<10$ & $N$ & 70 & $<5$ & .5 & 21 & $<.001$ & 1.20 \\
\hline LIII144 & 200 & $N$ & 5 & 15 & $N$ & $N$ & $\langle 100$ & 15 & $N$ & $N$ & 100 & $<5$ & .4 & 8 & $<.001$ & .7 \\
\hline Lili145 & 200 & $N$ & 5 & 20 & $<5$ & $N$ & $<100$ & 20 & $\langle 10$ & $N$ & 100 & $<5$ & .4 & 10 & $<.001$ & .70 \\
\hline LINI46 & 300 & $N$ & 10 & 30 & 5 & $N$ & 100 & 30 & 10 & $N$ & 100 & $<5$ & .5 & 19 & $<.001$ & .7 \\
\hline LIMI47 & 300 & $N$ & 10 & 20 & 5 & $N$ & 150 & 50 & 15 & $N$ & 200 & $<5$ & .6 & 29 & $<.001$ & 1.00 \\
\hline LIM148 & 100 & $N$ & 5 & $<10$ & $N$ & $N$ & $\langle 100$ & 20 & $N$ & $N$ & 700 & 7 & .2 & 12 & $<.001$ & .6 \\
\hline LIKI49 & 70 & $N$ & 5 & $N$ & $N$ & $N$ & $N$ & 15 & $N$ & $N$ & 500 & 8 & .1 & 7 & $<.001$ & \\
\hline
\end{tabular}

Nillion Hills--Continued

\begin{tabular}{|c|c|c|c|c|c|c|c|c|c|c|c|c|c|c|c|c|}
\hline MILO44 & 150 & $N$ & 5 & 15 & $N$ & $N$ & $N$ & 15 & $<10$ & $N$ & 70 & $<5$ & .3 & 8 & $<.001$ & .65 \\
\hline HIL045 & 700 & $N$ & 20 & 30 & 7 & $N$ & $<100$ & 50 & 20 & $N$ & 300 & 5 & 1.2 & 37 & .001 & 1.70 \\
\hline HIL150 & 1,000 & $<20$ & 70 & 20 & 15 & $N$ & 300 & 100 & 30 & $<200$ & 200 & $<5$ & 1.8 & 47 & .001 & 1.10 \\
\hline HILI51 & 2,000 & $<20$ & 150 & 30 & 30 & $N$ & 100 & 500 & 150 & 200 & 700 & 17 & 5.6 & 56 & .001 & 1.90 \\
\hline KIL152 & 1,500 & $<20$ & 70 & 30 & 10 & $N$ & $<100$ & 200 & 20 & $<200$ & 500 & 11 & 3.6 & 37 & .001 & 1.50 \\
\hline KIL153 & 700 & $N$ & 20 & 30 & 7 & $N$ & 200 & 50 & 15 & $N$ & 200 & $<5$ & 1.1 & 37 & $<.001$ & 1.30 \\
\hline KILI54 & 700 & $N$ & 30 & 20 & 7 & $N$ & 100 & 70 & 20 & $N$ & 150 & 12 & 1.6 & 37 & .001 & 1.70 \\
\hline KIL155 & 300 & $N$ & 50 & 20 & 10 & $N$ & 200 & 100 & 20 & $N$ & 150 & 12 & 1.4 & 43 & .002 & 1.50 \\
\hline MIL156 & 1.000 & $<20$ & 70 & 30 & 15 & $<10$ & 200 & 150 & 30 & $<200$ & 500 & 6 & 2.4 & 46 & .001 & 1.00 \\
\hline RILI57 & 200 & $N$ & 10 & 20 & $N$ & $N$ & $<100$ & 20 & 30 & $N$ & 200 & 5 & .5 & 19 & $<.001$ & .55 \\
\hline HILI58 & 150 & $N$ & 10 & 15 & $N$ & $N$ & $<100$ & 20 & $<10$ & $N$ & 300 & $<5$ & .4 & 10 & $<.001$ & .45 \\
\hline KILI59 & 200 & $N$ & 15 & 10 & 5 & N & $<100$ & 70 & 10 & $N$ & 500 & 5 & .5 & 6 & $<.001$ & .65 \\
\hline MILI60 & 100 & N & $<5$ & $N$ & $N$ & $N$ & $N$ & 15 & $N$ & $N$ & 200 & $<5$ & .2 & 4 & $<.001$ & .50 \\
\hline HIL161 & 100 & $N$ & $<5$ & $<10$ & $N$ & $N$ & $N$ & 20 & $N$ & $N$ & 150 & $<5$ & .2 & $<2$ & $<.001$ & .45 \\
\hline HILI62 & 1.000 & $<20$ & 70 & 30 & 20 & $N$ & 100 & 100 & 70 & $<200$ & 500 & $<5$ & 1.3 & 48 & .001 & 1.50 \\
\hline MIL163 & 1.000 & $<20$ & 200 & 30 & 20 & $<10$ & 300 & 150 & 50 & $<200$ & 500 & $<5$ & 2.0 & 56 & .003 & 2.00 \\
\hline NILI64 & 700 & $N$ & 20 & 30 & 7 & $N$ & 200 & 70 & 30 & $N$ & 300 & 6 & 1.0 & 37 & $<.001$ & 1.90 \\
\hline HILI65 & 500 & $N$ & 50 & 20 & 10 & $N$ & 200 & 70 & 20 & $N$ & 500 & 8 & 1.7 & 42 & .001 & 2.40 \\
\hline MILI66 & 300 & $N$ & 20 & 20 & 7 & N & $<100$ & 50 & 15 & N & 300 & 6 & .6 & 32 & .001 & 1.70 \\
\hline HIL167 & 500 & $N$ & 30 & 15 & 7 & $N$ & 150 & 100 & 20 & $N$ & 300 & 5 & 1.3 & 40 & $<.001$ & 1.00 \\
\hline KiL168 & 300 & $\mathrm{~N}$ & 20 & 20 & 5 & $N$ & 100 & 100 & 20 & $N$ & 300 & 5 & 1.1 & 10 & $<.001$ & .70 \\
\hline MILI69 & 500 & $N$ & 20 & 20 & 7 & N & 100 & 70 & 15 & N & 200 & 9 & 1.4 & 31 & .001 & 1.10 \\
\hline MILI70 & 1.000 & N & 50 & 20 & 15 & $\langle 10$ & 200 & 100 & 50 & $<200$ & 150 & 8 & 2.7 & 44 & .001 & 1.60 \\
\hline KIL171 & 200 & $N$ & 15 & 30 & $<5$ & $N$ & $<100$ & 30 & 15 & $N$ & 300 & $<5$ & .7 & 24 & $<.001$ & .70 \\
\hline
\end{tabular}


TABLE 4.--Results of analyses of streau-sediment samples frow the Liwe Canyon and Million Hills Hilderaess Study Areas, Clark County, Nevada--Continued

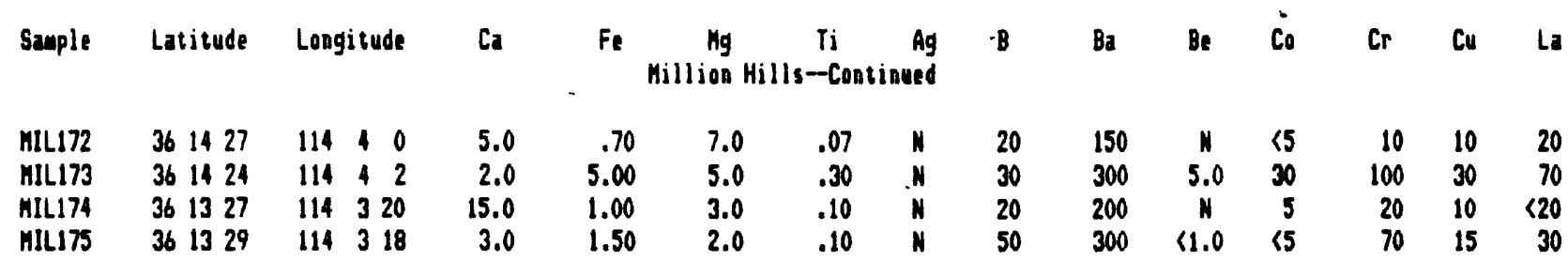

TABLE 4.--Results of analyses of strean-sediuent samples from the Lime Canyon and Hillion Hills Hilderaess Study Areas, Clark County, Meuada--Continued

\begin{tabular}{|c|c|c|c|c|c|c|c|c|c|c|c|c|c|c|c|c|}
\hline Sauple & $\mathrm{Mn}$ & $\mathrm{NH}$ & $\mathrm{Ni}$ & $\mathrm{Pb}$ & Sc & $\mathrm{Sn}_{\mathrm{n}}$ & $\begin{array}{l}\text { Sr } \\
\text { llion }\end{array}$ & $\begin{array}{c}v \\
\text { ills- }\end{array}$ & $\begin{array}{l}y \\
\text { atiou }\end{array}$ & $2 n$ & $2 r$ & $A_{5}-i$ & $C+-i$ & $2 n-i$ & Au-a & $U-f$ \\
\hline IIL172 & 200 & $N$ & 10 & 15 & $N$ & $N$ & $\langle 100$ & 50 & $<10$ & N & 100 & 6 & .5 & 9 & $<.001$ & \\
\hline ILI73 & 1.000 & N & 70 & 20 & 20 & $<10$ & 200 & 150 & 50 & $\angle 200$ & 300 & $<5$ & 1.7 & 37 & .001 & 9 \\
\hline LI74 & 200 & N & 5 & 20 & $<5$ & $N$ & $\langle 100$ & 30 & 15 & N & 300 & 5 & .4 & 12 & $(.001$ & \\
\hline 11175 & 300 & N & 10 & 15 & $<5$ & $N$ & 100 & 50 & 10 & N & 150 & 10 & .8 & 22 & $<.001$ & \\
\hline
\end{tabular}


TÁBLE 5.--Results of analyses of nonmadnetic heavy-mineral-concentrate sauples from the Lime Canyon and Million Hills Hilderness Study Areas, Clark County. Nevada

[N, not detected; 〈, detected below limit of determination shown: ), greater than upper limit of determination; --- not enough sample for analysis. Analyses by emission spectrography. Concentrations in ppm except Ca, Fe, Mg, Na, P, and $T i$, which are weight percent]

\begin{tabular}{|c|c|c|c|c|c|c|c|c|c|c|c|c|c|c|c|c|}
\hline Sauple & Latitude & Longitude & $\mathrm{Ca}$ & $\mathrm{Fe}$ & Mg & $\begin{array}{r}\mathrm{Na} \\
\text { Lime }\end{array}$ & $\begin{array}{r}P \\
\text { nyon }\end{array}$ & $\mathrm{Ti}$ & Ag & $B$ & $\mathrm{Ba}$ & $\mathrm{Be}$ & $\mathrm{Bi}$ & Co & $\mathrm{Cr}$ & Cu \\
\hline LIM033H & 361558 & 114168 & 5.0 & 1.50 & 1.50 & .5 & .5 & 2.00 & $N$ & N & 1,000 & N & N & $N$ & N & $<10$ \\
\hline LIMO34H & $\begin{array}{lll}36 & 16 \quad 4\end{array}$ & 1141634 & 5.0 & .70 & .50 & $<.5$ & 5.0 & .30 & N & 20 & 1,000 & $<2$ & $N$ & $N$ & $N$ & 10 \\
\hline LInO35H & 361625 & 114177 & 7.0 & 1.00 & .30 & .5 & 5.0 & .20 & $N$ & $<20$ & 300 & $N$ & N & $N$ & $<20$ & $<10$ \\
\hline LIMO36H & 361648 & 1141839 & 2.0 & 1.00 & .50 & $N$ & .7 & 1.00 & N & $N$ & 150 & N & N & N & $<20$ & \\
\hline LIM037H & 361725 & 114208 & 2.0 & .50 & .50 & $<.5$ & 1.0 & 1.00 & N & N & 150 & 2 & N & N & N & \\
\hline LIMO38H & 361728 & 114207 & 5.0 & 1.00 & 2.00 & N & 10.0 & 1.50 & N & $N$ & 200 & $N$ & $N$ & N & $<20$ & \\
\hline LIM039H & $\begin{array}{lll}36 & 18 & 0\end{array}$ & 1141936 & 3.0 & 1.50 & 1.00 & N & 1.0 & 2.00 & N & N & 200 & $N$ & N & N & N & 10 \\
\hline LIMO4OH & 361811 & 1141844 & 5.0 & .70 & 7.00 & $N$ & 3.0 & .70 & $N$ & N & 200 & $N$ & N & N & $<20$ & \\
\hline LIM04IH & 361711 & 1141923 & 3.0 & .20 & .50 & $N$ & 3.0 & .30 & N & $N$ & 500 & $N$ & $N$ & $N$ & N & \\
\hline LIMO42H & 361650 & 1142027 & 3.0 & .20 & .20 & $N$ & 5.0 & .30 & N & N & 200 & N & N & N & N & \\
\hline LIM043H & 361623 & 114200 & 2.0 & .30 & .20 & N & 5.0 & .50 & N & $N$ & 100 & $N$ & N & N & N & \\
\hline LIM101H & 361754 & $114 \quad 1313$ & 2.0 & .20 & .30 & N & 2.0 & .70 & N & N & 200 & N & N & $N$ & N & 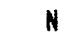 \\
\hline LIMIO2H & 361824 & $114 \quad 14 \quad 8$ & 1.0 & .70 & 5.00 & N & 1.0 & 1.00 & $N$ & $N$ & 700 & $N$ & N & N & N & W \\
\hline LIM103H & 361825 & $114 \quad 14 \quad 13$ & 5.0 & .30 & 1.50 & N & 3.0 & 1.00 & N & $N$ & 5.000 & N & N & $N$ & $N$ & \\
\hline LIMIO4H & 362051 & 1141412 & 2.0 & .70 & 2.00 & N & $<.5$ & .50 & $N$ & $N$ & $>10,000$ & $N$ & $<20$ & $N$ & N & 15 \\
\hline LIMI05H & 362050 & 1141416 & 2.0 & .15 & 2.00 & N & .7 & 1.00 & N & N & 1,500 & N & $N$ & N & 20 & II \\
\hline LIM106H & 362130 & 1141433 & 1.5 & 1.50 & 1.00 & N & 1.0 & 2.00 & $N$ & 70 & 5,000 & 3 & N & $N$ & 30 & 50 \\
\hline LIMIOTH & 362153 & 1141418 & .3 & 2.00 & .50 & N & $N$ & 1.00 & N & 30 & 5,000 & $<2$ & $N$ & $<20$ & 50 & $<10$ \\
\hline LIMI08H & 362220 & 1141424 & 1.5 & .70 & .70 & N & .5 & 1.50 & $N$ & $<20$ & 10,000 & $N$ & N & N & $<20$ & \\
\hline LIM109H & $\begin{array}{lll}36 & 23 & 7\end{array}$ & 114144 & 2.0 & .70 & .50 & N & 2.0 & 2.00 & $N$ & 20 & 7,000 & 2 & $N$ & N & 30 & $N$ \\
\hline LInIIOH & 362350 & 1.141353 & 1.5 & .50 & .70 & N & 1.0 & 1.50 & $N$ & $<20$ & 10,000 & $<2$ & N & N & 20 & \\
\hline LIMILH & 362413 & 1141344 & 3.0 & .70 & .30 & N & 3.0 & 1.00 & $N$ & 20 & 7,000 & $<2$ & N & $N$ & 30 & 10 \\
\hline LIMII2H & 362450 & 1141356 & 2.0 & .70 & 2.00 & N & $N$ & .07 & $N$ & $N$ & 10.000 & $N$ & $<20$ & N & N & $<10$ \\
\hline LIMII3H & 362554 & 1141346 & 3.0 & .70 & .30 & $<.5$ & .7 & .50 & N & N & $>10,000$ & 2 & N & N & N & $<10$ \\
\hline LIMIIIH & 362645 & 1141415 & .3 & .20 & .05 & $<.5$ & N & .10 & $N$ & N & $>10.000$ & $N$ & $<20$ & $N$ & N & $<10$ \\
\hline LIMI15H & 53 & $11416 \quad 1$ & .5 & .30 & .15 & $N$ & $N$ & .20 & $N$ & $N$ & .000 & $N$ & $<20$ & $N$ & $N$ & 120 \\
\hline LIMII6H & 362628 & 1141650 & 1.0 & .30 & .70 & N & $<.5$ & .03 & $N$ & $N$ & $>10.000$ & $N$ & $<20$ & N & N & $<10$ \\
\hline LIMI17H & 36263 & 1141639 & 1.5 & .50 & 1.00 & N & .5 & 1.00 & $N$ & 30 & $>10,000$ & $N$ & N & N & 20 & 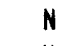 \\
\hline LIMI18H & 362530 & 1141624 & .7 & 2.00 & 1.00 & N & N & 1.50 & $N$ & 50 & $>10,000$ & $<2$ & $N$ & $N$ & 100 & 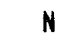 \\
\hline LIM119H & $3625 \quad 2$ & 1141622 & 1.0 & .50 & 1.50 & N & N & .50 & $N$ & N & $>10,000$ & $N$ & $<20$ & N & N & $<10$ \\
\hline LIM120H & 362427 & 1141719 & 5.0 & .30 & 5.00 & N & $<.5$ & .70 & $N$ & $<20$ & $>10,000$ & N & $N$ & N & N & \\
\hline LIM12IH & 36237 & 1141726 & 2.0 & 1.00 & 2.00 & N & .7 & 1.00 & $N$ & 30 & 5,000 & $<2$ & N & N & 30 & $<10$ \\
\hline LIM122H & 362238 & 114175 & 5.0 & .50 & 10.00 & N & .5 & .50 & $N$ & $N$ & 3,000 & $N$ & N & N & $<20$ & 15 \\
\hline LIM123H & 36226 & 1141820 & .7 & .70 & .30 & N & $N$ & .10 & $N$ & $N$ & $>10,000$ & N & N & N & N & $<10$ \\
\hline LIM124H & 362210 & 1141745 & 5.0 & 1.00 & 5.00 & N & $<.5$ & .70 & $N$ & $<20$ & 7,000 & N & N & N & N & 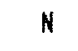 \\
\hline LIMI25H & $36 \quad 2233$ & $114 \quad 18 \quad 3$ & 3.0 & 2.00 & 7.00 & $N$ & $N$ & .50 & $n$ & 30 & 5,000 & $\mathbf{N}$ & $N$ & N & 200 & 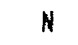 \\
\hline LIM126H & 362141 & $114 \quad 18 \quad 17$ & 1.5 & 1.50 & 1.00 & $N$ & <.5 & 1.00 & $N$ & $N$ & $>10.000$ & $N$ & $N$ & N & $<20$ & 10 \\
\hline LIMI27H & 362147 & 1141847 & 5.0 & .50 & 5.00 & $N$ & 3.0 & .70 & H & $N$ & $>10,000$ & $N$ & N & N & $<20$ & 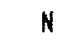 \\
\hline LIM128H & 362039 & 1141911 & 7.0 & .50 & 7.00 & $N$ & .7 & .70 & $N$ & $N$ & 5.000 & $<2$ & $N$ & $N$ & $N$ & N \\
\hline LIMI29H & 361938 & $114 \quad 1831$ & 3.0 & .20 & 3.00 & $N$ & .7 & .70 & $N$ & 20 & $>10,000$ & $N$ & $N$ & $N$ & $N$ & \\
\hline
\end{tabular}


TABLE 5.--Results of analyses of nonmagnetic heauy-wineral-concentrate samples from the Lime Canyon and Million Hills Hilderness Study Areas, Clark County. Nevada--Continued

\begin{tabular}{|c|c|c|c|c|c|c|c|c|c|c|c|c|c|c|c|c|}
\hline Sample & $\mathrm{Ga}$ & La & $\mathrm{Mn}$ & Mo & $\mathrm{Nb}$ & $\mathrm{Ni}$ & $\begin{array}{c}\mathrm{Pb} \\
\text { Liue Car }\end{array}$ & $\begin{array}{c}\mathrm{Sc} \\
\text { yon-- }\end{array}$ & $\begin{array}{c}5 n \\
\text { ontin }\end{array}$ & Sr & Th & v & $H$ & Y & Zn & $\mathrm{Zr}$ \\
\hline LIH033H & $<10$ & 300 & 200 & $N$ & $<50$ & $\leqslant 10$ & 200 & $<10$ & 30 & N & 700 & 100 & $N$ & 500 & $N$ & $>2,000$ \\
\hline LIM034H & $N$ & 200 & 150 & $N$ & $N$ & $N$ & 100 & 10 & $N$ & $N$ & $N$ & 20 & $<50$ & 500 & $N$ & 22,000 \\
\hline LIM035H & $N$ & 200 & 200 & $N$ & $N$ & $<10$ & 500 & N & $N$ & N & $N$ & 150 & $N$ & 300 & N & $>2.000$ \\
\hline LIM036H & N & 300 & 100 & $N$ & N & $N$ & 20 & $<10$ & $N$ & $N$ & $N$ & 30 & $N$ & 300 & N & $\lambda 2,000$ \\
\hline LIH037H & N & 200 & 150 & $N$ & $<50$ & N & 30 & $<10$ & $N$ & $N$ & $N$ & 30 & N & 150 & N & $>2,000$ \\
\hline LIM038H & N & 500 & 500 & $N$ & $<50$ & $N$ & 30 & 15 & N & N & N & 50 & $N$ & 700 & $N$ & 32,000 \\
\hline LIMO39H & $N$ & 500 & 200 & $N$ & $<50$ & $N$ & $<20$ & $<10$ & $N$ & N & $N$ & 50 & $N$ & 500 & $N$ & 22,000 \\
\hline LIMO4OH & $N$ & 300 & 150 & $N$ & N & $<10$ & 20 & $<10$ & $N$ & $N$ & N & 30 & $<50$ & 300 & $N$ & $>2,000$ \\
\hline LIMO4IH & $N$ & 150 & 30 & $N$ & N & $N$ & 20 & $N$ & N & $<200$ & $N$ & 30 & $N$ & 200 & $N$ & $>2.000$ \\
\hline LIM042H & $N$ & 150 & 30 & $N$ & $\mathbf{N}$ & N & 20 & $<10$ & N & $<200$ & N & 50 & N & 200 & $N$ & $>2,000$ \\
\hline LIM043H & N & 150 & 20 & $N$ & $N$ & $N$ & 20 & $N$ & $N$ & $<200$ & $N$ & 50 & $N$ & 200 & N & 32,000 \\
\hline LIM101H & $N$ & 100 & 30 & $N$ & $N$ & N & $<20$ & $<10$ & N & $<200$ & $N$ & 30 & N & 300 & $N$ & $>2,000$ \\
\hline LIM102H & $N$ & 300 & 150 & $<10$ & $<50$ & $N$ & 70 & $N$ & $N$ & $N$ & N & 50 & 100 & 300 & $N$ & 32,000 \\
\hline LIM103H & $N$ & 100 & 50 & $N$ & $N$ & $N$ & $<20$ & $<10$ & $N$ & $N$ & $N$ & 50 & $N$ & 300 & $N$ & 22,000 \\
\hline LIHIO4H & N & $<100$ & 30 & 15 & $N$ & $N$ & 200 & N & $N$ & $>10,000$ & $N$ & 70 & 300 & 100 & N & $>2,000$ \\
\hline LIM105H & $N$ & $N$ & $<20$ & $N$ & $N$ & $N$ & $N$ & $<10$ & $N$ & $<200$ & $N$ & 30 & $N$ & 200 & $N$ & $>2,000$ \\
\hline LIM106H & $N$ & 150 & 50 & $N$ & $<50$ & $<10$ & 100 & 10 & $N$ & 300 & $N$ & 150 & N & 500 & $N$ & $>2,000$ \\
\hline LIn107H & $N$ & 300 & 500 & $N$ & $N$ & 10 & $<20$ & $<10$ & $N$ & $<200$ & $N$ & 100 & $N$ & 300 & $N$ & 22,000 \\
\hline LIMIO8H & N & 150 & $<20$ & $N$ & N & $N$ & $N$ & 10 & N & 200 & $N$ & 70 & N & 500 & $N$ & 72,000 \\
\hline LIM109H & N & $<100$ & 20 & $N$ & $N$ & N & $<20$ & 15 & $N$ & $N$ & $N$ & 100 & N & 500 & $N$ & $>2,000$ \\
\hline LIMI1OH & $N$ & 100 & 30 & $<10$ & N & $N$ & $N$ & 15 & $N$ & $<200$ & N & 70 & 200 & 500 & $N$ & $>2,000$ \\
\hline LIH1LH & $N$ & 100 & 30 & N & N & N & $<20$ & $<10$ & N & $<200$ & N & 70 & $N$ & 300 & N & 22,000 \\
\hline LIMI12H & $N$ & $N$ & 20 & $N$ & $N$ & N & 100 & $N$ & $N$ & $>10,000$ & $N$ & 20 & $N$ & $N$ & $N$ & 700 \\
\hline LIMI13H & $N$ & $<100$ & 100 & N & $<50$ & $N$ & 30 & $N$ & $N$ & $>10,000$ & $N$ & 50 & $N$ & 70 & $N$ & $>2,000$ \\
\hline LIMI14H & $N$ & $N$ & 70 & $N$ & $N$ & $N$ & $<20$ & $N$ & $N$ & $>10,000$ & $N$ & 20 & $N$ & $N$ & $N$ & 1,500 \\
\hline LIM115H & $N$ & $<100$ & 20 & $N$ & $N$ & $N$ & 20 & $N$ & $N$ & $>10,000$ & $N$ & 70 & $N$ & 30 & $N$ & $>2,000$ \\
\hline LIH116H & $N$ & $N$ & $<20$ & $N$ & $N$ & $N$ & $N$ & $N$ & N & $>10,000$ & $N$ & 70 & $N$ & $<20$ & $N$ & 2,000 \\
\hline LIM117H & $N$ & $N$ & $<20$ & $N$ & $N$ & $<10$ & 70 & $<10$ & N & $>10,000$ & $N$ & 50 & $N$ & 200 & $N$ & $>2,000$ \\
\hline LIMI18H & $N$ & 150 & 100 & $N$ & $<50$ & 15 & $<20$ & 10 & N & 3,000 & $N$ & 100 & $N$ & 300 & $N$ & $>2,000$ \\
\hline LInII9H & $N$ & $N$ & $<20$ & $N$ & $N$ & N & $N$ & $N$ & N & 7,000 & $N$ & 70 & $N$ & 150 & $N$ & $\lambda 2,000$ \\
\hline LIM12OH & $N$ & 150 & 30 & $N$ & $N$ & $N$ & $<20$ & $N$ & $N$ & 300 & $N$ & 50 & $N$ & 200 & $N$ & 32,000 \\
\hline LIM12IH & $N$ & 100 & 50 & $N$ & $N$ & $N$ & $<20$ & $<10$ & $<20$ & $<200$ & $N$ & 100 & $N$ & 300 & $N$ & $>2,000$ \\
\hline LIM122H & $N$ & $N$ & 20 & $N$ & $N$ & $N$ & 100 & $N$ & $N$ & $N$ & $N$ & 100 & $N$ & 150 & $N$ & $>2,000$ \\
\hline LIM123H & $N$ & $<100$ & 30 & $N$ & $N$ & $N$ & 2,000 & $N$ & N & $>10,000$ & $N$ & 100 & $N$ & 20 & $N$ & 22,000 \\
\hline LIM124H & $N$ & $N$ & $<20$ & $N$ & $N$ & $N$ & 50 & $N$ & $N$ & $<200$ & $N$ & 70 & $N$ & 150 & $N$ & 12,000 \\
\hline LIM125H & $N$ & $N$ & 30 & $N$ & $N$ & $<10$ & $<20$ & $N$ & $N$ & 300 & $N$ & 70 & $N$ & 100 & $N$ & $>2,000$ \\
\hline LIM126H & $N$ & $N$ & 50 & $N$ & $N$ & $<10$ & 500 & $<10$ & $N$ & 10,000 & $N$ & 100 & $N$ & 200 & 1.500 & $>2,000$ \\
\hline LIM127H & N & 150 & 70 & $N$ & $<50$ & $N$ & $<20$ & $<10$ & $N$ & 300 & $N$ & 50 & $N$ & 200 & $N$ & $>2,000$ \\
\hline LIM128H & N & 100 & 70 & $N$ & $<50$ & $N$ & 20 & $<10$ & $N$ & $<200$ & $N$ & 50 & $<50$ & 200 & $N$ & 12,000 \\
\hline LIH129H & N & $N$ & 30 & $N$ & $N$ & $N$ & $<20$ & $N$ & $N$ & $>10,000$ & $N$ & 70 & $N$ & 150 & $N$ & 32,000 \\
\hline
\end{tabular}


TABLE 5.--Results of analyses of nonmagnetic heauy-mineral-concentrate samples from the Lime Canyon and Million Hills Uilderness Study Areas, Clark County, Nevada--Continued

\begin{tabular}{|c|c|c|c|c|c|c|c|c|c|c|c|c|c|c|c|c|}
\hline Sample & Latitude & Longitude & $\mathrm{Ca}$ & $\mathrm{Fe}$ & $\begin{array}{l}\text { Mg } \\
\text { Lime }\end{array}$ & $\mathrm{Na}$ & $\stackrel{P}{P}$ & $\mathrm{Ti}$ & ASG & B & $\mathrm{Ba}$ & $\mathrm{Be}$ & $\mathrm{Bi}$ & Co & $\mathrm{Cr}$ & Cu \\
\hline LIM130H & 361926 & $114 \quad 1743$ & 1.5 & .10 & .50 & N & 1.0 & .15 & $N$ & N & $>10,000$ & N & $N$ & N & $N$ & \\
\hline LIM131H & 361913 & $114 \quad 1821$ & 7.0 & .50 & 2.00 & .5 & 1.0 & .50 & N & $N$ & 2,000 & N & $N$ & $\mathbf{N}$ & $<20$ & 10 \\
\hline LIMI32H & $36 \quad 1855$ & 1141823 & 10.0 & .30 & 5.00 & $N$ & 7.0 & .50 & $N$ & $<20$ & 1,000 & $N$ & $<20$ & N & $<20$ & 10 \\
\hline LIM133H & $36 \quad 1841$ & 1141836 & 7.0 & .50 & 7.00 & N & 1.5 & 1.50 & $N$ & 200 & 1.000 & $N$ & $\mathbf{N}$ & $N$ & 100 & 10 \\
\hline LIM134H & $36 \quad 16 \quad 13$ & 1141527 & 2.0 & .70 & .70 & N & .5 & 1.50 & N & N & 3,000 & N & N & N & 20 & \\
\hline LIMI35H & $36 \quad 16 \quad 18$ & 1141521 & 5.0 & .30 & 5.00 & $N$ & 1.5 & .70 & N & $<20$ & 500 & $N$ & N & N & 20 & 10 \\
\hline LIMI36H & 361557 & $11415 \quad 1$ & 2.0 & 1.00 & 1.00 & $N$ & .7 & 1.50 & N & 20 & 1.500 & N & N & $N$ & 30 & 10 \\
\hline LIM137H & 361551 & 1141415 & 10.0 & .70 & .50 & $N$ & 10.0 & .70 & N & $N$ & 300 & N & N & N & 20 & 10 \\
\hline LIM138H & $36 \quad 1543$ & 1142037 & 5.0 & .50 & .10 & N & 5.0 & .70 & $N$ & $N$ & 700 & $N$ & $N$ & N & $N$ & \\
\hline LIM139H & 361847 & 1141434 & 3.0 & .30 & 1.50 & $N$ & 1.5 & .50 & N & $<20$ & 5,000 & 3 & $<20$ & $N$ & $<20$ & 10 \\
\hline Ini4OH & 361840 & 114 & 5.0 & .5 & 1.50 & $N$ & .7 & 2.00 & $N$ & 30 & 10.000 & $<2$ & N & $N$ & 30 & $<10$ \\
\hline LIMI4IH & $36 \quad 19 \quad 14$ & 1141556 & 5.0 & .50 & 5.00 & $N$ & $<.5$ & .70 & $<1$ & N & 5,000 & N & N & N & $N$ & \\
\hline LIH142H & 361922 & 1141623 & 1.5 & .50 & .20 & $N$ & .5 & .70 & $N$ & N & $>10,000$ & $N$ & $N$ & $N$ & $<20$ & \\
\hline LIM143H & 361925 & 1141616 & 7.0 & 1.50 & 5.00 & $N$ & 2.0 & 1.00 & $<1$ & 20 & 7,000 & $<2$ & N & $N$ & 30 & 3 \\
\hline LIMI44H & 36190 & 1141531 & 7.0 & 1.00 & 10.00 & N & $<.5$ & .50 & $N$ & N & $>10,000$ & N & $N$ & N & $<20$ & ve \\
\hline LIMI45H & $\begin{array}{lll}36 & 19 & 1\end{array}$ & 1141545 & --- & -- & --- & --- & -- & -- & --- & --- & --- &.-- & --- & -- & --- & 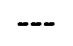 \\
\hline LIM146H & 361952 & 1141659 & 10.0 & .30 & 3.00 & N & 2.0 & .70 & N & N & 2,000 & N & $N$ & $N$ & $<20$ & 3 \\
\hline LIM147H & 361951 & 1141653 & 5.0 & .50 & 2.00 & N & 1.0 & 1.00 & N & N & 1,500 & N & $N$ & N & 20 & \\
\hline LIMI 48H & $36 \quad 2331$ & 114157 & 1.5 & .70 & .20 & N & .7 & 1.00 & $N$ & 50 & $>10,000$ & N & N & $N$ & 70 & \\
\hline LIM149H & $36 \quad 23 \quad 35$ & $114 \quad 15 \quad 5$ & 1.0 & .20 & .10 & N & .5 & .15 & N & $N$ & 5,000 & $N$ & $N$ & $\mathrm{~N}$ & 20 & \\
\hline
\end{tabular}

Million Hills

\begin{tabular}{|c|c|c|c|c|c|c|c|c|c|c|c|c|c|c|c|c|c|}
\hline MILO44H & 361910 & 114 & 520 & 7.0 & .50 & 10.00 & N & .7 & .07 & 5 & $N$ & 150 & $N$ & $<20$ & N & $N$ & $<10$ \\
\hline MILO45H & 361910 & 114 & 521 & 7.0 & .50 & 7.00 & $N$ & 3.0 & .70 & $N$ & $N$ & 1,500 & 2 & 20 & N & 20 & $N$ \\
\hline MILI5OH & $36 \quad 1852$ & 114 & 330 & 10.0 & .50 & 1.00 & $<.5$ & 7.0 & .20 & $N$ & $\mathrm{~N}$ & 10,000 & $N$ & $N$ & N & $<20$ & 20 \\
\hline MILI51H & 361935 & 114 & 258 & 3.0 & .50 & .70 & N & 1.0 & .70 & $N$ & $N$ & 2,000 & $<2$ & $N$ & N & $<20$ & 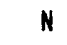 \\
\hline MILL152H & $36 \quad 1858$ & 114 & 222 & 5.0 & .30 & 1.50 & N & 1.0 & .70 & $N$ & $N$ & 300 & $N$ & N & N & 20 & \\
\hline MILI53H & $36 \quad 2031$ & 114 & 250 & 10.0 & .50 & 1.00 & N & 7.0 & .70 & $N$ & $N$ & 500 & N & $\mathrm{N}$ & N & 20 & 30 \\
\hline MIL154H & $36 \quad 2033$ & 114 & 247 & 7.0 & .10 & 1.00 & $N$ & 7.0 & .50 & $N$ & $N$ & 3,000 & N & N & N & $N$ & 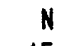 \\
\hline MILI55H & $36 \quad 1947$ & 114 & 232 & 10.0 & 1.00 & 2.00 & $<.5$ & 5.0 & .70 & $N$ & $N$ & 1,000 & 2 & $\mathbf{N}$ & N & 20 & 15 \\
\hline MILL156H & 361955 & 114 & 235 & 5.0 & .50 & 1.00 & N & 2.0 & .20 & $N$ & N & 100 & N & N & M & $N$ & 15 \\
\hline MIL157H & $36 \quad 1736$ & 114 & 234 & 5.0 & 1.00 & 5.00 & $N$ & 1.0 & 1.00 & $N$ & 20 & 300 & N & N & $\mathbb{N}$ & 200 & \\
\hline MILI58H & 361741 & 114 & 231 & 10.0 & 1.50 & 3.00 & $N$ & 2.0 & 1.00 & $\mathbf{N}$ & 20 & 500 & N & $N$ & N & 150 & \\
\hline MIL159H & $36 \quad 16 \quad 12$ & 114 & 336 & 2.0 & .70 & 2.00 & H & 1.0 & .30 & $N$ & N & 10,000 & N & $N$ & N & 20 & \\
\hline MIL160H & $36 \quad 16 \quad 14$ & 114 & 338 & 1.5 & .50 & .50 & N & .7 & .70 & $N$ & $<20$ & $>10,000$ & N & $N$ & N & 200 & \\
\hline MIL161H & 361616 & 114 & 336 & 5.0 & .20 & 5.00 & N & 1.0 & .30 & $N$ & N & 10,000 & N & N & N & 50 & \\
\hline MILI62H & 36155 & 114 & 58 & 5.0 & .70 & 1.50 & .5 & 1.0 & .30 & $N$ & N & 1,500 & N & N & N & $<20$ & \\
\hline MIL163H & $\begin{array}{lll}36 & 15 & 3\end{array}$ & 114 & $5 i$ & 7.0 & .10 & .50 & N & 7.0 & .05 & $N$ & N & 500 & N & N & N & N & 10 \\
\hline MIL164H & 36155 & 114 & 25 & 7.0 & .70 & 1.00 & N & 5.0 & .50 & $N$ & N & 1,000 & $N$ & $N$ & N & 30 & 10 \\
\hline MIL165H & 361539 & 114 & 146 & 7.0 & .20 & .20 & N & 5.0 & .15 & $N$ & N & 200 & N & N & N & $N$ & $<10$ \\
\hline MIL166H & $\begin{array}{lll}36 & 17 & 0\end{array}$ & 114 & 214 & 5.0 & .30 & 2.00 & $N$ & .7 & 1.00 & $N$ & $N$ & 310,000 & N & $N$ & $\mathrm{~N}$ & $<20$ & \\
\hline MIL167H & $36155 t$ & 114 & 21 & 7.0 & .20 & 1.00 & $n$ & 1.5 & .20 & $N$ & N & 1.500 & 50 & $N$ & N & N & 1 \\
\hline MIL168H & $36 \quad 160$ & 114 & 157 & 5.0 & .15 & 5.00 & N & 1.0 & .50 & $N$ & N & 2,000 & N & $N$ & N & $N$ & \\
\hline MIL169H & 361556 & 114 & 159 & 7.0 & .30 & 2.00 & $N$ & 5.0 & .50 & $N$ & N & 2,000 & N & N & $N$ & N & 2 \\
\hline MIL170H & 36143 & 114 & 312 & 10.0 & .15 & .70 & $N$ & 7.0 & .30 & $N$ & N & 1,500 & $N$ & N & N & N & 1 \\
\hline MIL171H & 361416 & 114 & 340 & 3.0 & .50 & 1.50 & $<.5$ & 1.5 & 1.50 & $\mathrm{H}$ & 20 & 150 & $N$ & N & N & 50 & \\
\hline
\end{tabular}


TABLE 5.--Fesults of analyses of nommanetic heauy-mineral-concentrate samples from the Lime Canyon and Million Hills Hilderness Study Areas, Clark County, Nevada--Continued

\begin{tabular}{|c|c|c|c|c|c|c|c|c|c|c|c|c|c|c|c|c|}
\hline Sauple & Ga & $\mathrm{La}$ & Mn & Mo & $\mathrm{Nb}$ & $\mathrm{Ni}$ & $\begin{array}{l}\mathrm{Pb} \\
\text { Line } \mathrm{Ca}\end{array}$ & Sc & $\begin{array}{c}\mathrm{Sn} \\
\text { intin }\end{array}$ & $\mathrm{Sr}$ & Th & v & $H$ & Y & $2 n$ & $2 r$ \\
\hline LIM130H & $N$ & $<100$ & 20 & 10 & N & $N$ & $<20$ & $<10$ & $N$ & $>10,000$ & N & 50 & 300 & 150 & $N$ & $>2,000$ \\
\hline LIM131H & N & 200 & 50 & $\mathrm{~N}$ & $N$ & $\langle 10$ & 20 & $N$ & N & $\langle 200$ & N & 30 & N & 150 & $\mathrm{~N}$ & $>2,000$ \\
\hline LIMI32H & N & 150 & 70 & $N$ & $N$ & $<10$ & $\ll 20$ & $N$ & $N$ & $<200$ & N & 50 & $N$ & 150 & $N$ & $>2,000$ \\
\hline LIMI33H & $\mathrm{N}$ & 100 & 50 & $\mathrm{~N}$ & 50 & N & 20 & N & 70 & N & $\mathrm{N}$ & 150 & 50 & 100 & $\mathrm{~N}$ & $>2,000$ \\
\hline LIM134H & N & $<100$ & 20 & N & N & 15 & $<20$ & $<10$ & $N$ & $<200$ & N & 50 & $N$ & 200 & $N$ & 32,000 \\
\hline LIM135H & N & 150 & 30 & N & N & $<10$ & 300 & N & N & $<200$ & 5,000 & 100 & N & 200 & $N$ & $>2,000$ \\
\hline LIMI36H & N & 200 & 150 & N & $<50$ & 30 & 150 & $N$ & N & $<200$ & 1,000 & 70 & $<50$ & 300 & $N$ & 32,000 \\
\hline LIM137H & $<10$ & 1,000 & 200 & $N$ & $N$ & $<10$ & 30 & $<10$ & N & $\langle 200$ & N & 50 & $N$ & 1,000 & $N$ & $>2,000$ \\
\hline LIN138H & $N$ & 200 & 150 & N & N & $N$ & 50 & $N$ & $N$ & N & $<200$ & 50 & $N$ & 300 & $N$ & 72,000 \\
\hline LIM139H & $N$ & 150 & 50 & $\cdot<10$ & $N$ & N & 30 & $N$ & $N$ & $<200$ & $N$ & 30 & 100 & 200 & $N$ & $>2,000$ \\
\hline LIM140H & N & $<100$ & 20 & N & N & $N$ & 200 & 10 & $N$ & 10,000 & N & 70 & $N$ & 500 & 3,000 & $>2,000$ \\
\hline LIMI41H & $N$ & N & 30 & N & N & $N$ & 30 & $<10$ & 30 & $<200$ & $N$ & 50 & $N$ & 200 & $<500$ & $>2,000$ \\
\hline LIMI42H & $N$ & $<100$ & 50 & N & $N$ & N & $<20$ & N & $N$ & 5,000 & $N$ & 30 & $<50$ & 200 & $N$ & 22,000 \\
\hline LIM143H & $<10$ & 150 & 50 & $N$ & N & $<10$ & 1,000 & $<10$ & 70 & $<200$ & $N$ & 150 & $N$ & 300 & $<500$ & $>2,000$ \\
\hline LIMI44H & N & $N$ & 50 & $N$ & N & N & 200 & N & $N$ & $N$ & $N$ & 70 & $N$ & 100 & $N$ & 32,000 \\
\hline LIM145H & -- & $\ldots$ & - & - & -- & -- & -- & --- & --- & -- & -- & -- & -- & - & - & -- \\
\hline LIM146H & $N$ & 100 & 20 & N & $N$ & $N$ & 300 & $<10$ & $<20$ & 200 & $N$ & 200 & $N$ & 300 & $N$ & 72,000 \\
\hline LIM147h & $N$ & $<100$ & 50 & $N$ & $N$ & $N$ & 20 & $<10$ & N & N & $N$ & 50 & N & 150 & $N$ & 72,000 \\
\hline LIM148H & $N$ & $<100$ & $<20$ & N & $N$ & $N$ & $<20$ & $<10$ & N & 300 & $N$ & 50 & $N$ & 200 & $N$ & 72,000 \\
\hline LIM149H & $N$ & $N$ & $<20$ & N & $N$ & $N$ & $<20$ & $N$ & $N$ & 200 & $N$ & 20 & $N$ & 50 & $N$ & $>2,000$ \\
\hline
\end{tabular}

\section{Hillion Hills--Continued}

\begin{tabular}{|c|c|c|c|c|c|c|c|c|c|c|c|c|c|c|c|c|}
\hline MILO44H & $N$ & $N$ & $<20$ & N & $N$ & $N$ & 3,000 & N & $N$ & $N$ & N & 150 & 200 & 50 & N & 2,000 \\
\hline HILO45H & N & 100 & 70 & $<10$ & $N$ & $N$ & 20 & $\langle 10$ & $N$ & $\langle 200$ & N & 100 & 300 & 200 & $N$ & $>2,000$ \\
\hline MILI5OH & $N$ & 300 & 150 & $<10$ & $N$ & $<10$ & 500 & $N$ & N & $<200$ & $N$ & 20 & 200 & 300 & $<500$ & 22,000 \\
\hline MILI5IH & N & 200 & 50 & N & N & $N$ & 30 & $<10$ & $N$ & $N$ & $N$ & 30 & N & 200 & $N$ & 32,000 \\
\hline MIL152H & $N$ & 100 & 20 & 10 & $N$ & N & 70 & $<10$ & N & N & $N$ & 50 & 100 & 150 & $N$ & 32,000 \\
\hline MIL153H & N & 500 & 200 & $N$ & $N$ & $N$ & 50 & $N$ & N & $<200$ & $N$ & 50 & $N$ & 200 & N & $>2,000$ \\
\hline MIL154H & N & 150 & 100 & N & N & N & $<20$ & $<10$ & N & $N$ & $N$ & 20 & N & 200 & $N$ & $>2,000$ \\
\hline MIL155H & $N$ & 500 & 300 & N & $N$ & $<10$ & 20 & 10 & $N$ & $<200$ & $N$ & 70 & N & 500 & N & $>2,000$ \\
\hline MIL156H & N & 200 & 100 & N & N & N & 20 & $N$ & N & N & N & 50 & N & 150 & N & 32,000 \\
\hline MiL157H & $N$ & 150 & 30 & N & N & $<10$ & $<20$ & $<10$ & N & $N$ & N & 70 & N & 300 & $N$ & 32,000 \\
\hline MIL158H & N & 150 & 150 & N & $N$ & $<10$ & 50 & $N$ & $N$ & $N$ & N & 70 & N & 200 & N & 32,000 \\
\hline MILI59H & N & 300 & 70 & N & $N$ & N & 30 & $<10$ & $N$ & $<200$ & $N$ & 30 & 100 & 150 & N & $>2,000$ \\
\hline MIL16OH & $N$ & $N$ & 1,000 & N & N & N & $<20$ & $<10$ & $N$ & 200 & N & 50 & 70 & 500 & $N$ & 12,000 \\
\hline MIL16IH & N & $N$ & 300 & N & $N$ & N & $N$ & N & N & N & N & 50 & N & 100 & $N$ & $>2,000$ \\
\hline MIL162H & 10 & 150 & 300 & 50 & $\$ 50$ & N & $<20$ & N & N & N & N & 30 & 1,000 & 150 & $N$ & 2,000 \\
\hline MIL163H & N & 100 & N & N & $N$ & N & $<20$ & $N$ & N & $<200$ & N & 20 & N & 100 & $N$ & $>2,000$ \\
\hline MILI64H & N & 150 & 300 & $N$ & $N$ & N & $\prec 20$ & N & $N$ & $<200$ & $N$ & 50 & N & 200 & $N$ & $>2,000$ \\
\hline MIL165H & N & 100 & 30 & N & $N$ & N & 50 & N & N & $\langle 200$ & 200 & 100 & N & 150 & $N$ & $>2,000$ \\
\hline MIL166H & N & 100 & 500 & N & $N$ & N & $<20$ & $N$ & N & 200 & $N$ & 50 & N & 150 & $N$ & $>2,000$ \\
\hline MIL167H & N & 150 & 70 & N & $N$ & $N$ & 20 & $<10$ & $N$ & $<200-$ & N & 20 & N & 200 & $N$ & $>2,000$ \\
\hline MILI68H & $N$ & $<100$ & 300 & $N$ & N & N & N & $<10$ & N & $N$ & N & 20 & $N$ & 150 & $N$ & $>2,000$ \\
\hline MIL169H & $N$ & 300 & 300 & $N$ & $N$ & N & 30 & $<10$ & N & $<200$ & $N$ & 50 & $N$ & 200 & $N$ & $>2,000$ \\
\hline MIL170H & $N$ & $500^{\circ}$ & 150 & $N$ & $N$ & n & 50 & $\langle 10$ & N & $<200$ & 200 & 50 & $<50$ & 500 & $N$ & $>2.000$ \\
\hline MIL171H & $N$ & $<100$ & 1,000 & $N$ & $N$ & $N$ & $<20$ & $<10$ & N & $N$ & N & 70 & N & 300 & $N$ & $>2,000$ \\
\hline
\end{tabular}


TABLE 5.--Results of analyses of nonmagnetic heauy-uineral-concentrate sauples frow the Liwe Canyon and Hillion Hills Hilderness Study Areas, Clark County, Mevada--Contiaued

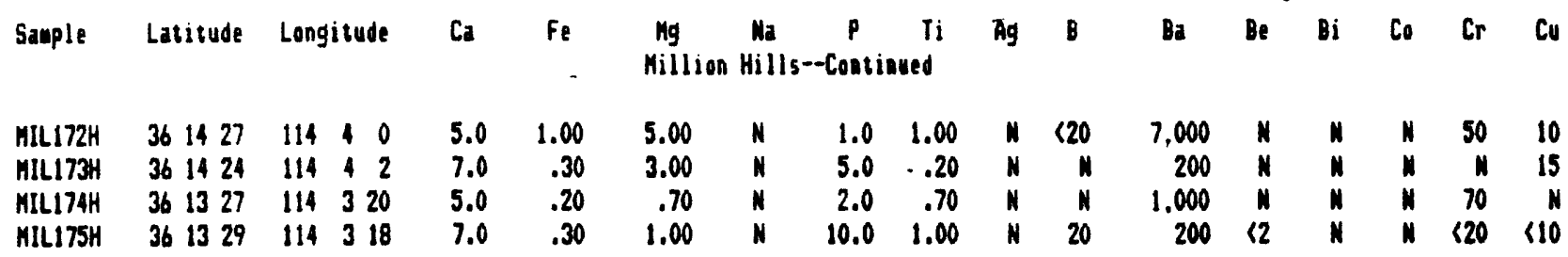

TABLE 5.--Results of analyses of nonmagnetic heauy-nineral-concentrate samples from the Lime Canyon and Hillion Hills Hilderaess Study Areas, Clark County, Nevada--Contiaued

\begin{tabular}{|c|c|c|c|c|c|c|c|c|c|c|c|c|c|c|c|c|}
\hline Sample & Ga & La & Min & Mo & NH & $\mathrm{Ni}$ & & $\begin{array}{r}\text { So } \\
\text { lills }\end{array}$ & $S_{n}$ & $\mathrm{sr}$ & Th & $v$ & $H$ & $Y$ & 20 & $2 r$ \\
\hline HILI72H & $N$ & 200 & 1,000 & $n$ & $N$ & $N$ & $<20$ & $<10$ & N & $N$ & $N$ & 70 & $N$ & 300 & $N$ & 22,000 \\
\hline MILI73H & $N$ & 200 & 150 & $N$ & $N$ & $N$ & $<20$ & $N$ & $N$ & $<200$ & $N$ & 30 & $N$ & 150 & $N$ & 32,000 \\
\hline MIL174H & $N$ & $<100$ & 700 & $n$ & $H$ & $<10$ & N & $<10$ & $N$ & $N$ & $N$ & 50 & $N$ & 300 & N & 22,000 \\
\hline - HIL175H & $N$ & 300 & 150 & $N$ & $N$ & N & 50 & $<10$ & N & $<200$ & $<200$ & 50 & $<50$ & 300 & $N$ & 22,000 \\
\hline
\end{tabular}


TABBLE 6. Results of analyses of raw panned-concentrate samples frow the Liwe Canyon and Million Hills Hilderness Study Areas, Clark County, Nevada

[<, less than value shown. Au-a in ppw. Height, graws of raw parined-concentrate sauple. Analyses by atomic absorption]

\begin{tabular}{|c|c|c|c|c|}
\hline Sauple & Latitude & $\begin{array}{l}\text { Longitude } \\
\text { we Canyor }\end{array}$ & $A u-a$ & Weight \\
\hline LIM033G & 361558 & $11416 \quad 8$ & $<.01$ & 60.57 \\
\hline LIM034G & $36 \quad 164$ & 1141634 & $<.03$ & 20.45 \\
\hline LIM035G & $36 \quad 16 \quad 25$ & $114 \quad 17 \quad 7$ & .10 & 72.01 \\
\hline LIM036G & 361648 & 1141839 & $<.06$ & 8.46 \\
\hline LIM037G & $36 \quad 1725$ & 114208 & $<.01$ & 67.61 \\
\hline LIM038G & $36 \quad 1728$ & 114207 & $<.03$ & 18.03 \\
\hline LIM039G & 36180 & 1141936 & $<.02$ & 35.96 \\
\hline LIMO40G & 361811 & 1141844 & $<.02$ & 33.14 \\
\hline LIM041G & 361711 & 1141923 & .02 & 53.27 \\
\hline LIMO42G & 361650 & 1142027 & .02 & 39.06 \\
\hline LIM043G & 361623 & 114200 & $<.04$ & 14.09 \\
\hline LIMIOIG & $36 \quad 1754$ & 1141313 & $<.03$ & 23.46 \\
\hline LIM102G & $36 \quad 18 \quad 24$ & 114148 & $<.03$ & 23.23 \\
\hline LIMI03E & $36 \quad 1825$ & 1141413 & $<.03$ & 23.90 \\
\hline LInt04G & 362051 & 1141412 & $<.03$ & 21.54 \\
\hline LIM105G & 362050 & 1141416 & $<.30$ & 1.67 \\
\hline LIM106G & $36 \quad 2130$ & $114 \quad 1433$ & $<.18$ & 2.87 \\
\hline LIM107G & $36 \quad 2153$ & 1141418 & .65 & 4.48 \\
\hline LIMI080 & $3622 \quad 20$ & 1141424 & $<.10$ & 5.55 \\
\hline LIMI09G & 36237 & $11414 \quad 4$ & $<.23$ & 2.25 \\
\hline LIM110G & 362350 & 1141353 & $<.12$ & 4.17 \\
\hline LIM111G & $36 \quad 24 \quad 13$ & 1141344 & $<.20$ & 2.61 \\
\hline LIMI12G & 362450 & 1141356 & $\langle .21$ & 2.48 \\
\hline LIM1130 & $36 \quad 2554$ & 1141346 & $<.11$ & 4.95 \\
\hline LIMI14G & 362645 & 1141415 & $<.06$ & 8.35 \\
\hline LIM1150 & $36 \quad 26 \quad 53$ & 114161 & $<.04$ & 12.60 \\
\hline LIMHLG & $36 \quad 26 \quad 28$ & 1141650 & .47 & 10.96 \\
\hline LIMIITG & $\begin{array}{lll}36 & 26 & 3\end{array}$ & 1141639 & $<.35$ & 1.45 \\
\hline LIM118G & 362530 & 1141624 & $<.14$ & 3.82 \\
\hline LIMI19G & $\begin{array}{lll}36 & 25 & 2\end{array}$ & 1141622 & $<.22$ & 2.38 \\
\hline LIMI20G & $36 \quad 2427$ & 1141719 & $<.04$ & 14.42 \\
\hline LIM1216 & $\begin{array}{lll}36 & 23 & 7\end{array}$ & 1141726 & $<.14$ & 3.63 \\
\hline LIHI22G & $36 \quad 2238$ & $114 \quad 17 \quad 5$ & $<.16$ & 3.16 \\
\hline LIM1236 & 36226 & 1141820 & $<.05$ & 11.07 \\
\hline LIM1246 & $36 \quad 22 \quad 10$ & 1141745 & .33 & 2.13 \\
\hline LIM125G & $36 \quad 2233$ & 114183 & $<.20$ & 2.51 \\
\hline LIMI26G & 362141 & $114 \quad 18 \quad 17$ & $<.38$ & 1.34 \\
\hline LIM1276 & $\begin{array}{lll}36 & 21 \quad 47\end{array}$ & 1141847 & $<.02$ & 37.13 \\
\hline LIM128G & 362039 & 1141911 & $<.05$ & 11.86 \\
\hline LIM129G & $36 \quad 1938$ & 1141831 & $<.08$ & 6.41 \\
\hline
\end{tabular}


TABLE 6.--Results of amalyses of raw panaed-concentrate samples from the Lime Cayon and Hillion Hills Hilderaess Study Areas, Clark Couaty, Mevada--Coup̧iaued

\begin{tabular}{|c|c|c|c|c|}
\hline Sauple & $\begin{array}{l}\text { Latitude } \\
\text { - Live Cal }\end{array}$ & $\begin{array}{l}\text { Longitude } \\
\text { yon--Contin }\end{array}$ & $A v-a$ & Height \\
\hline $\begin{array}{l}\text { LIM130G } \\
\text { LIM1310 } \\
\text { LIM1326 } \\
\text { LIM1330 } \\
\text { LIn134G }\end{array}$ & $\begin{array}{lll}36 & 19 & 26 \\
36 & 19 & 13 \\
36 & 18 & 55 \\
36 & 18 & 11 \\
36 & 16 & 13\end{array}$ & $\begin{array}{lll}114 & 17 & 13 \\
114 & 18 & 21 \\
114 & 18 & 23 \\
114 & 18 & 36 \\
114 & 15 & 27\end{array}$ & $\begin{array}{l}<.02 \\
<.02 \\
<.02 \\
.04 \\
<.02\end{array}$ & $\begin{array}{l}39.82 \\
40.26 \\
40.59 \\
39.75 \\
49.73\end{array}$ \\
\hline $\begin{array}{l}\text { LIM1356 } \\
\text { LIn1366 } \\
\text { LIM1376 } \\
\text { LIM1380 } \\
\text { LIMI39G }\end{array}$ & $\begin{array}{llll}36 & 16 & 18 \\
36 & 15 & 57 \\
36 & 15 & 51 \\
36 & 15 & 13 \\
36 & 18 & 17\end{array}$ & $\begin{array}{lll}114 & 15 & 21 \\
114 & 15 & 1 \\
114 & 14 & 15 \\
114 & 20 & 37 \\
114 & 14 & 34\end{array}$ & $\begin{array}{l}<.02 \\
<.01 \\
<.03 \\
<.04 \\
C .04\end{array}$ & $\begin{array}{l}48.99 \\
50.70 \\
23.86 \\
12.59 \\
14.48\end{array}$ \\
\hline $\begin{array}{l}\text { LIM140G } \\
\text { LIM1416 } \\
\text { LIM1426 } \\
\text { LIMI436 } \\
\text { LIm1446 }\end{array}$ & $\begin{array}{lll}36 & 18 & 40 \\
36 & 19 & 14 \\
36 & 19 & 22 \\
36 & 19 & 25 \\
36 & 19 & 0\end{array}$ & $\begin{array}{lll}114 & 14 & 36 \\
114 & 15 & 56 \\
114 & 16 & 23 \\
114 & 16 & 16 \\
114 & 15 & 31\end{array}$ & $\begin{array}{l}<.09 \\
<.17 \\
<.02 \\
<.23 \\
<.09\end{array}$ & $\begin{array}{r}5.55 \\
2.92 \\
45.43 \\
2.17 \\
5.80\end{array}$ \\
\hline $\begin{array}{l}\text { LIn1456 } \\
\text { LIn1460 } \\
\text { LIn1470 } \\
\text { LIn1486 } \\
\text { LIM1496 }\end{array}$ & $\begin{array}{lll}36 & 19 & 1 \\
36 & 19 & 52 \\
36 & 19 & 51 \\
36 & 23 & 31 \\
36 & 23 & 35\end{array}$ & $\begin{array}{lll}114 & 15 & 45 \\
114 & 16 & 59 \\
114 & 16 & 53 \\
114 & 15 & 7 \\
114 & 15 & 5\end{array}$ & $\begin{array}{l}<.09 \\
<.13 \\
<.16 \\
<.19 \\
<.24\end{array}$ & $\begin{array}{l}6.15 \\
4.01 \\
3.21 \\
2.79 \\
2.08\end{array}$ \\
\hline & & Hion Hills & & \\
\hline $\begin{array}{l}\text { MILO44G } \\
\text { MIL0456 } \\
\text { MILI506 } \\
\text { MIL151G }\end{array}$ & $\begin{array}{lll}36 & 19 & 10 \\
36 & 19 & 10 \\
36 & 18 & 52 \\
36 & 19 & 35\end{array}$ & $\begin{array}{lll}114 & 5 & 20 \\
114 & 5 & 21 \\
114 & 3 & 30 \\
114 & 2 & 58\end{array}$ & $\begin{array}{r}<.08 \\
<.02 \\
<.02 \\
.11\end{array}$ & $\begin{array}{r}6.57 \\
39.86 \\
25.53 \\
23.38\end{array}$ \\
\hline $\begin{array}{l}\text { niL1526 } \\
\text { KIL1530 } \\
\text { nIL1540 } \\
\text { nIL1556 } \\
\text { nIL1566 }\end{array}$ & $\begin{array}{lll}36 & 18 & 58 \\
36 & 20 & 31 \\
36 & 20 & 33 \\
36 & 19 & 17 \\
36 & 19 & 55\end{array}$ & $\begin{array}{lll}114 & 2 & 22 \\
114 & 2 & 50 \\
114 & 2 & 47 \\
114 & 2 & 32 \\
114 & 2 & 35\end{array}$ & $\begin{array}{l}.02 \\
<.05 \\
<.03 \\
<.05 \\
<.02\end{array}$ & $\begin{array}{r}26.59 \\
11.56 \\
17.85 \\
8.66 \\
37.05\end{array}$ \\
\hline $\begin{array}{l}\text { MILL1570 } \\
\text { HIL1586 } \\
\text { HIL1596 } \\
\text { HIL1600 } \\
\text { HIL1616 }\end{array}$ & $\begin{array}{lll}36 & 17 & 36 \\
36 & 17 & 11 \\
36 & 16 & 12 \\
36 & 16 & 14 \\
36 & 16 & 16\end{array}$ & $\begin{array}{lll}114 & 2 & 34 \\
114 & 2 & 31 \\
114 & 3 & 36 \\
114 & 3 & 38 \\
114 & 3 & 36\end{array}$ & $\begin{array}{l}<.07 \\
<.13 \\
1.02 \\
1.08 \\
1.09\end{array}$ & $\begin{array}{r}7.70 \\
3.91 \\
42.62 \\
6.53 \\
5.56\end{array}$ \\
\hline $\begin{array}{l}\text { MILI620 } \\
\text { MIL1630 } \\
\text { MILI640 } \\
\text { HIL1650 } \\
\text { MIL1660 }\end{array}$ & $\begin{array}{rrr}36 & 15 & 5 \\
36 & 15 & 3 \\
36 & 15 & 5 \\
36 & 15 & 39 \\
36 & 17 & 0\end{array}$ & $\begin{array}{lll}114 & 5 & 8 \\
114 & 5 & 7 \\
114 & 2 & 5 \\
114 & 1 & 46 \\
114 & 2 & 14\end{array}$ & $\begin{array}{l}.07 \\
.03 \\
<.02 \\
<.02 \\
.35\end{array}$ & $\begin{array}{l}29.75 \\
20.48 \\
29.94 \\
35.03 \\
12.93\end{array}$ \\
\hline $\begin{array}{l}\text { MILI670 } \\
\text { MILI680 } \\
\text { HILI690 } \\
\text { HILI706 } \\
\text { MILI716 }\end{array}$ & $\begin{array}{rrr}36 & 15 & 51 \\
36 & 16 & 0 \\
36 & 15 & 56 \\
36 & 14 & 3 \\
36 & 14 & 16\end{array}$ & $\begin{array}{lll}114 & 2 & 1 \\
114 & 1 & 57 \\
114 & 1 & 59 \\
114 & 3 & 12 \\
114 & 3 & 40\end{array}$ & $\begin{array}{l}<.11 \\
<.02 \\
<.01 \\
r .02 \\
<.11\end{array}$ & $\begin{array}{r}4.37 \\
39.48 \\
51.14 \\
48.21 \\
4.41\end{array}$ \\
\hline $\begin{array}{l}\text { MILI726 } \\
\text { MILI73G } \\
\text { BILI746 } \\
\text { HIL1756 }\end{array}$ & $\begin{array}{lll}36 & 14 & 27 \\
36 & 14 & 24 \\
36 & 13 & 27 \\
36 & 13 & 29\end{array}$ & $\begin{array}{lll}114 & 4 & 0 \\
114 & 4 & 2 \\
114 & 3 & 20 \\
114 & 3 & 18\end{array}$ & $\begin{array}{l}<.07 \\
1.07 \\
8.15 \\
1.10\end{array}$ & $\begin{array}{l}7.00 \\
7.68 \\
3.34 \\
5.04\end{array}$ \\
\hline
\end{tabular}

